\title{
TRANSPORT THEORY OF INTERACTING QUANTUM DOTS
}

\author{
H. SCHOELLER \\ Institut für Theoretische Festkörperphysik, \\ Universität Karlsruhe, \\ 76128 Karlsruhe, Germany
}

\section{Introduction}

In the last few years the progress in microfabrication technology has led to an enhanced interest in transport properties of ultrasmall conducting islands coupled weakly to leads (for reviews see Refs.[1-6]). Quantization of charge and tunneling through zero-dimensional states lead to many interesting phenomena in these systems. Adding a single charge to a small island costs the charging energy $E_{C} \sim e^{2} /(\epsilon L) \sim e^{2} /(2 C)$ ( $L$ being the length scale of the island, $\epsilon$ the dielectric constant, and $C$ the self-capacitance), and, second, the level spacing $\delta E$ of the single-particle states. For system lengths in the nanoscale regime, charging energies can be reached of order $1-10 \mathrm{~K}$. For temperatures below $1 \mathrm{~K}$ this implies that electron transport can be completely blocked (Coulomb blockade) or being restricted to a small number of possible charge states. In the same way electron transport can be influenced by the discrete level structure on the island. Especially in $2 \mathrm{~d}$ semiconductor quantum dots the level spacing is large (typically $1 / 10$ of the charging energy).

The sensitivity to adding a single charge can be used for measurement applications, e.g. for the detection of single charges or for setting up current standards. Electronic applications are the subject of intensive research and could become of technological interest if the operating temperature of these devices can be increased. Experimentalists can use single electron phenomena as spectroscopic tools. For theoreticians quantum dots are important systems for studying models of strongly correlated systems in equilibrium or nonequilibrium. Quantum dots represent various realizations of generalized Kondo and Anderson models. Arrays of quantum dots can be used to model Hubbard chains. 
Many phenomena in single electron devices can be understood within golden rule theory. This means that tunneling to the particle reservoirs is so weak that the spectral density of the island remains unchanged and transport can be described by classical master equations, the so-called orthodox theory [1]. A crucial assumption in justifying perturbation theory is a small intrinsic broadening of the island excitations compared to temperature $T$ (we always set $k_{B}=1$ ). Experimentally this can easily be achieved by using tunneling barriers with tunneling resistances $R_{T}$ much higher than the quantum resistance $R_{K}=h / e^{2}$. Thus, there exists a well-defined experimental regime where perturbation theory can describe single-electron tunneling through zero-dimensional states [2].

It is important to notice that a master equation with golden rule tunneling rates is a perturbative approach in the coupling to the reservoirs but not in the interaction within the island. Therefore, this approach has to be distinguished from the well-known scattering formalism [7] which can describe coherent transport through mesoscopic devices for arbitrary tunneling barriers and temperatures but is restricted to noninteracting systems. It is therefore very important to formulate theories which can interpolate between both limits. It is the purpose of this report to present a technique which is capabable of describing coherent transport through interacting islands.

There are several experimental motivations to study coherent transport through strongly interacting quantum dots. First of all there are regimes where sequential tunneling is exponentially suppressed. This happens in the Coulomb blockade regime where the current is dominated by higher order processes such as coherent "cotunneling" processes of electrons through several junctions [8]. In interference geometries where quantum dots are part of an Aharonov-Bohm ring, only higher order processes beyond sequential tunneling show a flux dependence and lead to Aharonov-Bohm oscillations [9]. Experiments can be performed in the limit where the tunneling barriers are so low that even the case of perfect transmission can be reached without destroying the effect of Coulomb blockade. This leads to a significant deviation from "orthodox theory" even in regimes where sequential tunneling contributes. The spectral density of the island will be strongly affected by the coupling to the leads, and the broadening of levels will approach temperature or level spacing upon continously increasing tunneling. In the presence of interactions the broadening can be a complicated function of energy, temperature and bias voltage. This induces strong renormalization effects of the levels and the system parameters. For quantum dots described by one degenerate low-lying level it can even lead to new resonances in the spectral density in the form of Kondo resonances. They show up in various anomalies in the differential conductance as function of the bias voltage. 
Quantum dots with continuous level spectra are, in the two charge-state approximation, equivalent to multichannel Kondo models. Again, this gives rise to anomalous temperature dependences of the conductance as function of gate or bias voltage. By varying the level spacing, level position or using multi-dot systems an enormous variety of interesting many-body systems can be realized. Their low-temperature scaling behaviour is still not known for most cases.

Here we are interested in the case where the transmission per channel of the barriers is still much less than unity so that a well-defined description via a tunneling Hamiltonian is justified. One should recognize that, for large channel number, this includes the possibility of total transmission being larger than unity. Experiments in this regime have recently been performed in metallic dots with clear signs for deviations from classical behaviour [10]. Furthermore, quantum fluctuations become visible by lowering the temperature. Especially vertical quantum dots [11], ultrasmall metallic particles [12] or molecules [13], are promising candidates for the observation of quantum fluctuations in the weak transmission limit at realistic temperatures.

The transport theory presented here is based on a recently developed real-time diagrammatic approach [14-17]. It is closely related to path-integral methods using the Feynman-Vernon technique [18] formulated in connection with dissipation $[19,20]$ or tunneling in metallic junctions $[5,21]$. The idea is to integrate out the reservoir degrees of freedom and to set up a formally exact kinetic equation for the reduced density matrix of the dot. The kernel of this integro-differential equation is represented as a sum over all irreducible diagrams and can be calculated in a systematic perturbation expansion in tunneling. In this way the strong correlations on the island are fully taken into account. Furthermore, the golden rule theory, which is reproduced by using the kernel in lowest order perturbation theory, can be systematically generalized to higher orders. We will formulate an approximation for an explicit calculation of the kernel which reproduces the Landauer-Büttiker theory in the noninteracting limit but provides also a good description for coherent transport in the strongly interacting case.

\section{Single-electron devices}

\subsection{MOTIVATION: THE COULOMB BLOCKADE MODEL}

In this section we discuss the basic physical properties of quantum dots. We introduce a simplified model and discuss the conditions for various energy scales when Coulomb blockade phenomena and tunneling through zero-dimensional states are observable.

We consider a small island containing interacting electrons in a uni- 


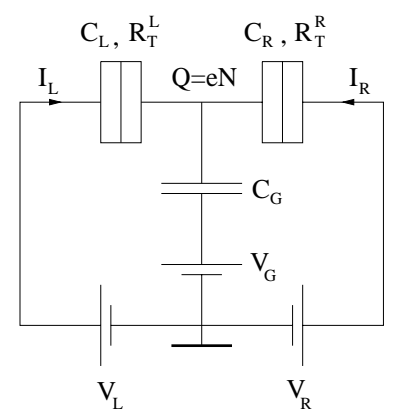

Figure 1. The SET transistor. All three terminals are coupled capacitively to the island. Two tunnel junctions allow transport from the left reservoir to the right one.

form positive background charge. The island is coupled electrostatically to macroscopic metallic reservoirs with different electrochemical potentials $\mu_{r}=e V_{r}, r=L, R$. A current can flow by tunneling of electrons across the tunnel junctions. A schematic view of such an arrangement is shown in Fig. 1. The total charge on the island is given by $Q=e N$, where $\mathrm{N}$ denotes the excess electron number. By means of a third terminal, called the gate, which is coupled electrostatically to the island, one can change the electrochemical potential of the island independent of $V_{L}$ and $V_{R}$. In this way it is possible to control the particle number on the island. Such a system is called a single-electron transistor (SET) in the general nonequilibrium situation where $V_{L} \neq V_{R}$, or a single electron box (SEB) for the equilibrium case where $V_{L}=V_{R}$.

The length scale $L$ of the island is typically of order $0.1-1 \mu \mathrm{m}$. This is large compared to atomic scales and it is possible to couple the island to macroscopic voltage sources. On the other hand, the system size is so small that single charge-transfer processes can be measured on a $m e V$ voltage scale. Adding one single charge to the neutral island will cost a charging energy $E_{C} \sim e^{2} /(\epsilon L) \sim 0.1-1 \mathrm{meV} \sim 1-10 \mathrm{~K}$ where we have assumed $\epsilon \sim 10$ for typical semiconductor quantum dots. The level spacing $\delta E \sim\left(k_{F} L\right)^{2-d} \hbar^{2} \pi^{2} /\left(m^{*} L^{2}\right)$ defines the second energy scale for adding one electron. Here, $k_{F}$ is the Fermi wave vector, $d$ the dimension, and $m^{*}$ the effective electron mass. To achieve $\delta E \sim 1 K$, one has to reduce the dimensionality or use smaller system sizes. For a $3 d$ metallic system with Fermi wave length $\lambda_{F} \sim 10 \AA$, one needs $L \sim 10 \mathrm{~nm}$. For a $2 \mathrm{~d}$ electron gas it is sufficient to take $L \sim 100 \mathrm{~nm}$. Furthermore, the level spacing is increased in systems with small effective mass.

We start with an analysis of the concept of charging energy. To calculate the electrostatic work $E_{\text {pot }}$ to build up an arbitrary charge distribution on the island we use the so-called Coulomb blockade model. It means that the 
island is treated like a metal, i.e., the electrostatic potential on the island is assumed to be homogeneous. For $3 d$ metallic systems this is usually a good approximation except for system sizes $L$ smaller than $10 \mathrm{~nm}$ where the Thomas-Fermi screening length $\lambda_{T F}=\left(\lambda_{F} a_{B} / 8\right)^{1 / 2}$ starts to become comparable to $L$ and the potential is no longer homogeneous over the island. In $2 \mathrm{~d}$ semiconductor quantum dots there is no exponential screening and the screening length is given by the Bohr radius $a_{B}$. Here it depends on the particle number and the distance to the gates whether the Coulomb blockade model can be used.

Within the capacitive model the electrostatic work $E_{\text {pot }}(Q)$ to build up the total charge $Q$ on the island is given by $E_{\text {pot }}(Q)=\int_{0}^{Q} d Q^{\prime} V\left(Q^{\prime}\right)$, where $V(Q)$ is the electrostatic potential of the island for given island charge $Q$. It depends on the fixed voltages $V_{i}, i=L, R, g$, of the metallic reservoirs and follows from $C_{i}\left(V_{i}-V\right)=Q_{i}$, where $Q_{i}$ is the screening charge on capacitor $i$ (see Fig. 1 for notations). Using $-Q=Q_{L}+Q_{R}+Q_{g}$ together with the definitions $C=C_{L}+C_{R}+C_{g}$ and $q_{x}=-e n_{x}=\sum_{i=L, R, g} C_{i} V_{i}$, we obtain $V(Q)=\left(Q+q_{x}\right) / C$ and thus

$$
E_{\text {pot }}(Q=e N)=E_{C}\left(N-n_{x}\right)^{2},
$$

where we have added the irrelevant constant $E_{C} n_{x}^{2}$. The charging energy $E_{C}$ is given by $E_{C}=e^{2} /(2 C)$. For typical lengths $L \sim 0.1-1 \mu m$ and a dielectric constant $\epsilon \sim 10$, the capacitance is of order $C \sim 10^{-16}-10^{-15} \mathrm{~F}$.

The system tries to minimize its electrostatic energy. Therefore, the integer particle number $N$ tends to be as close as possible to the continuous variable $n_{x}$. As a consequence, the particle number on the island can be controlled in discrete units by varying $n_{x}$ via the gate voltage $V_{g}$. For half-integer values of $n_{x}$, two adjacent particle numbers $N=n_{x} \pm 1 / 2$ lead to the same electrostatic energy and transport is possible. Away from the degeneracy points, transport is suppressed up to smearing due to temperature, bias voltage and quantum fluctuations. This is the phenomenon of Coulomb-blockade.

So far we have considered only the Coulomb interaction. The total energy $E$ of the island is given by

$$
E=\sum_{k} \epsilon_{k D} n_{k D}+E_{C}\left(N-n_{x}\right)^{2},
$$

where $\mid k D>$ are single-particle states of the dot with occupation $n_{k D}$ and energy $\epsilon_{k D} . k$ is the wave vector numerating the states. Furthermore, the total excess particle number is given by $N=\sum_{k} n_{k D}-N_{0}$, where $N_{0}$ is the number of electrons on the neutral island. If the particle number increases from $N$ to $N+1$, the ground state energy changes by the amount 


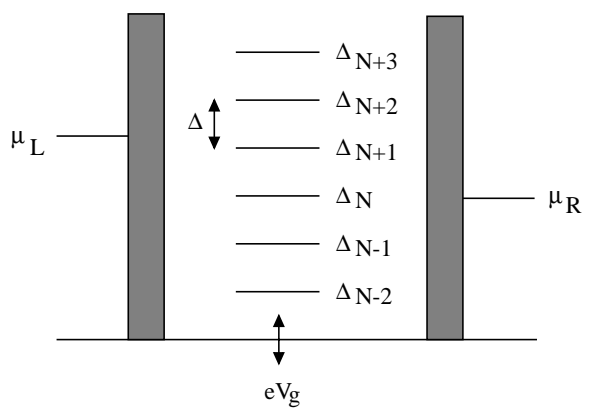

Figure 2. One-particle excitation energies of the Coulomb blockade model. For simplicity it is assumed that the level spacing is a constant. If an excitation $\Delta_{N}$ falls into the window of the electrochemical potentials of the reservoirsTtransport can occur. The position of $\Delta_{N}$ depends linearly on the gate voltage $V_{g}$.

$\Delta_{N}=E_{N+1}^{0}-E_{N}^{0}=\epsilon_{N+N_{0}+1}^{D}+2 E_{C}\left(N-n_{x}\right)+E_{C}$. It describes a oneparticle excitation energy of the island corresponding to a transition between ground state energies with different particle numbers. The quantity $\Delta_{N}$ can also be regarded as the definition of the electrochemical potential of the island. Of course there are other excitations involving excited states, which become important if the level spacing $\delta E$ is smaller than temperature or bias voltage.

We are now able to set up the conditions when transport is possible. In Fig. 2 we have shown an energy profile of the double barrier structure indicating all electrochemical potentials of the reservoirs and the excitation energies of the island. For constant level spacing $\delta E$, all excitation energies are equidistant $\Delta=\Delta_{N+1}-\Delta_{N}=\delta E+2 E_{C}$. Furthermore, their absolute position can be shifted linearly by the electrochemical potential $\mu_{g}=e V_{g}$ of the gate $\partial \Delta_{N} / \partial \mu_{g}=C_{g} / C$. In lowest order perturbation theory in the tunneling barriers, energy conservation and the Pauli principle restrict tunneling. This means that one of the excitations $\Delta_{N}$ has to lie within the window of the electrochemical potentials of the reservoirs $\mu_{R}<\Delta_{N}<\mu_{L}$. For finite temperatures, this condition has to be fulfilled only within the smearing defined by the Fermi distribution function. If no excitation lies between $\mu_{R}$ and $\mu_{L}$, transport is suppressed. Thus, in order to observe a significant modulation of the current as function of the gate voltage, we need $T, e V=e\left(V_{L}-V_{R}\right) \ll \Delta$ which implies $T, e V \ll \delta E$ or $T, e V \ll E_{C}$. The first condition guarantees transport through zero-dimensional states, whereas the second one implies the occurence of Coulomb-blockade phenomena.

Within golden rule theory it is sufficient to consider the excitation spectrum of the isolated dot as shown in Fig. 2. This means that we have neglected so far the fact that the spectral density of the dot itself can be 
changed by the presence of the reservoirs. Due to the finite life-time $\tau$ of the excitations there will be a corresponding broadening $\sim \hbar / \tau$ and via Kramers-Kronig also a renormalization. We denote the temperature where the renormalization becomes significant by $T_{K}$ and call it "Kondo temperature" since the model is similiar to Kondo and Anderson models. The broadening and renormalization has two important consequences. First, in the low-temperature region where $T<\hbar / \tau$ or $T<T_{K}$, golden rule theory breaks down, higher order processes become important and nonperturbative methods have to be applied. This is the region where quantum fluctuations are important but single-electron tunneling still persists. Secondly, if the broadening $\hbar / \tau$ approaches the spacing $\Delta$ of the excitations, single electron phenomena will no longer be visible. This is the regime of strong tunneling.

Let us start with the case of large level spacing $\delta E \gg T$. Although the life-time of an excitation involving many-body states is strongly influenced by interactions (see section 4.3), a rough estimate for the energy scale of the broadening can be obtained by comparing with the noninteracting case. A single state in a double barrier has a Breit-Wigner broadening, of the order , $\equiv|t|^{2} \delta E$, where $|t|^{2}$ is the transmission probability of a single barrier [22]. For the Kondo temperature $T_{K}$, no general estimate is possible since it depends on the spectrum of the dot (see section 4.3). As already stated above, deviations from golden rule theory occur in the low-temperature region $T<$, or $T<T_{K}$ (see section 4.2 and 4.3). The regime of strong tunneling $\hbar / \tau \sim \delta E$ cannot be achieved here since, for high tunneling barriers, $|t|^{2} \ll 1$, and consequently $\hbar / \tau \sim$, $\ll \delta E$.

For $3 d$ metallic systems, where the level spacing $\delta E$ is very small, the situation is more complicated. Here, tunneling can happen through many excited states and the broadening of the charge excitations turns out to be , multiplied with the number of available states for tunneling into or out of the island (see section 4.4) $\hbar / \tau \sim \alpha_{0} \max \left(\Delta_{N}, T, e V\right)$. Here,

$$
\alpha_{0}=\frac{1}{4 \pi^{2}} \frac{R_{K}}{R_{T}}=\frac{1}{4 \pi^{2}} Z|t|^{2} \sim Z \frac{\rho}{\delta E}
$$

is, up to a conventional factor $1 /\left(4 \pi^{2}\right)$, the dimensionless conductance of a single barrier, and $Z$ the number of transverse channels. $R_{K}=h / e^{2}$ is the quantum resistance and $G_{T}=1 / R_{T}=Z\left(e^{2} / h\right)|t|^{2}$ the tunneling conductance of a single barrier. For $\Delta_{N} \sim E_{C} \gg T, e V$, the broadening is given by $\hbar / \tau \sim \alpha_{0} E_{C} \sim h /\left(R_{T} C\right)$ which agrees with the classical relaxation time for a charge on a capacitor in a $R C$-circuit. Single electron phenomena persist if the broadening $\hbar / \tau$ is much less than the distance $\Delta \sim E_{C}$ between the excitations. This is fulfilled for $\alpha_{0} \ll 1$ or, equivalently, $Z$, $\ll \delta E$. In contrast to the case of large level spacing, this condition is not automatically fulfilled for large tunneling barriers. For large transverse channel 
number $Z, \alpha_{0}$ can be of order unity even if,$\ll \delta E$. This is the regime of strong tunneling where quantum fluctuations are enhanced by lowering the tunneling barriers. When the condition $\alpha_{0} \ll 1$ is fulfilled, single electron phenomena are visible, but, due to renormalization of charge excitations, golden rule theory again has to be improved in the low-temperature regime (see section 4.4).

\subsection{HAMILTONIAN AND CURRENT OPERATOR}

In this section we will set up the Hamiltonian and the current operator. We distinguish between two different cases: Quantum dots with discrete quantum states and metallic islands with a continuous single-particle spectrum. We use the convention $\hbar=k_{B}=1$ and $e<0$.

\subsubsection{Quantum dots}

We consider a small island coupled to several metallic reservoirs and to an external heat bath. The bath can be represented by an environment or by internal bosonic degrees of freedom like, e.g., phonons or plasmons. For the general theory we need no assumption for the island Hamiltonian and include the possibility that the voltages on the reservoirs are time-dependent. The coupling to the reservoirs includes an electrostatic interaction as well as tunneling of electrons through high barriers. Let us first state the obvious form of the Hamiltonian and the current operator. For the interested reader, the explicit derivations are presented at the end of this section.

The model Hamiltonian reads $H(t)=H_{0}+H_{T}(t)$ with $H_{0}=H_{R}+H_{B}+$ $H_{D}$. Here, $H_{R}, H_{B}$ and $H_{D}$ denote the Hamiltonians for the reservoirs, the heat bath, and the dot, respectively, and $H_{T}(t)$ describes the tunneling between dot and reservoirs. Explicitly, we have

$$
\begin{aligned}
H_{0} & =H_{R}+H_{B}+H_{D} \\
& =\sum_{r=L, R} \sum_{k} \epsilon_{k}^{r} a_{k r}^{\dagger} a_{k r}+\sum_{q} \omega_{q} b_{q}^{\dagger} b_{q}+\sum_{s} E_{s} \hat{P}_{s}, \\
H_{T}(t) & =\sum_{r=L, R} \sum_{k, s s^{\prime}} \bar{T}_{k, s s^{\prime}}^{r}(t) a_{k r}^{\dagger} \hat{P}_{s s^{\prime}} e^{-i \hat{\phi}}+(\text { h.c. }) .
\end{aligned}
$$

All terms have an obvious interpretation. $\mid k r>$ denote the single particle states in reservoir $r$ with energy $\epsilon_{k}^{r}, \omega_{q}$ are the frequency modes of the heat bath, $E_{s}$ are the energy eigenvalues of the many-body states $|s\rangle$ of the isolated $\operatorname{dot}$, and $\hat{P}_{s}=|s\rangle\langle s|$ is the projector on state $|s\rangle$. For the Coulomb blockade model (2), the states $\mid s>$ of the dot are specified by the set of all occupation numbers for the single particle states: $|s\rangle=\left|\left\{n_{k D}\right\}\right\rangle$. The more general notation is introduced here since we want to include cases where the states of the dot cannot be described by single particle states, see 
e.g. $[23,24,33]$. Furthermore, the states $\mid s>$ can represent charge states (see section 2.2.2.), spin states, or states of multiple dots. This allows a unified treatment of many possibilities.

The tunneling part (5) describes charge transfer processes where the tunneling matrix element $\bar{T}_{k, s s^{\prime}}^{r}$ corresponds to a transition of the dot state from $\left|s^{\prime}\right\rangle$ to $|s\rangle$. Therefore, we have introduced the operators $\hat{P}_{s s^{\prime}}=$ $\left|s><s^{\prime}\right|$. Due to particle number conservation, we have $\bar{T}_{k, s s^{\prime}}^{r}=0$ unless $N_{s}=N_{s^{\prime}}-1$, where $N_{s}$ is the particle number on the dot for state $|s\rangle$. The electrostatic interaction between dot and reservoirs is described by the effective time dependence

$$
\bar{T}_{k, s s^{\prime}}^{r}(t)=T_{k, s s^{\prime}}^{r}(t) e^{i e \int_{t_{0}}^{t} d t^{\prime} \bar{V}_{r}\left(t^{\prime}\right)},
$$

where $\bar{V}_{r}(t)=V_{r}(t)-V_{D}(t)$ is the change of the electrostatic energy of a particle entering reservoir $r . V_{r}(t)$ denotes the time-dependent voltage on reservoir $r$, and $V_{D}(t)$ is the spatial average of the external electrostatic potential taken over the dot. The part of the electrostatic interaction which remains for zero voltage on all reservoirs is included in $H_{D}$. E.g. for the Coulomb blockade model (2), we have $V_{D}(t)=-2 E_{C} n_{x}(t)$ whereas the part $E_{C} \hat{N}^{2}$ of the electrostatic interaction is included in $H_{D}$. A possible explicit time dependence of the tunneling matrix elements $T$ in (6) accounts for a modulation of the barriers.

Finally, the bosonic phase factor $\exp (-i \hat{\phi})$ in $(5)$ describes the energy exchange with the heat bath due to absorption or emission of bosonic modes. The linear bosonic field $\hat{\phi}$ is defined by $\hat{\phi}=i \sum_{q} g_{q} / \omega_{q}\left(b_{q}-b_{q}^{\dagger}\right)$, where $g_{q}$ is the coupling constant to the heat bath for mode $q$. This model has been used widely in the literature, either to describe optical phonons in semiconductor quantum dots [25] or voltage fluctuations in metallic systems [26]. In the latter case, the relation between the coupling constants $g_{q}$, their spectral function $J(\omega)$, and the impedance $Z(\omega)$ of the external circuit is given by $[26]$

$$
J(\omega)=\pi \sum_{q} g_{q}^{2} \delta\left(\omega-\omega_{q}\right)=e^{2} \omega \operatorname{Re} Z(\omega),
$$

where $\omega>0$, since the bosonic modes $\omega_{q}$ are all positive. For the special case of ohmic dissipation $J(\omega) \sim \omega$, we obtain the Caldeira-Leggett model [19]. For an extended discussion of various kinds of possible environments we refer the reader to Ref. [27].

The physical observable which can be measured experimentally is the current $I_{r}$ flowing in reservoir $r$. This current consists of two contributions: a tunneling current $I_{r}^{\text {tun }}(t)$ from electrons hopping on or off the island and a 
displacement current $I_{r}^{d i s}(t)=\frac{d}{d t} Q_{r}(t)$ arising from the change of the timedependent screening charge $Q_{r}(t)$ on reservoir $r$. Let us show how the latter can be calculated for the simplified Coulomb blockade model introduced in section 2.1. For given charge $Q(t)$ on the island and given potentials $V_{r}(t)$, $r=L, R, g$, on the reservoirs and the gate we get for the screening charge $Q_{r}=C_{r}\left(V_{r}-V\right)$ with $V=\left(Q+q_{x}\right) / C$ being the potential on the island. Inserting the definition $q_{x}=\sum_{r} C_{r} V_{r}$ and taking the time derivative we get for the displacement current

$$
I_{r}^{d i s}=C_{r}\left[\dot{V}_{r}-\frac{1}{C}\left(\dot{Q}+\sum_{r^{\prime}} C_{r^{\prime}} \dot{V}_{r^{\prime}}\right)\right]
$$

The time-derivative of the island charge $\dot{Q}=\sum_{r} I_{r}^{\text {tun }}$ is known after we have calculated the tunneling currents. Summing (8) over $r$ we find total current conservation $\sum_{r} I_{r}(t)=\sum_{r}\left[I_{r}^{d i s}(t)+I_{r}^{\text {tun }}(t)\right]=0$. The displacement currents are only important for the calculation of AC-currents since the time average of $I_{r}^{\text {dis }}$ is usually zero except for cases where $\int \dot{V}_{r} \neq 0$.

The tunneling current operator $\hat{I}_{r}^{\text {tun }}(t)$ is given by the time derivative of the particle number operator in reservoir $r, \hat{I}_{r}^{t u n}(t)=-e \frac{d}{d t} \hat{N}_{r}=$ $-i \epsilon\left[H(t), \hat{N}_{r}\right]$. Inserting for $H(t)$ from (4) and (5) we find

$$
\hat{I}_{r}^{t u n}(t)=i e \sum_{k, s s^{\prime}} \bar{T}_{k, s s^{\prime}}^{r}(t) a_{k r}^{\dagger} \hat{P}_{s s^{\prime}} e^{-i \hat{\phi}}+(h . c .),
$$

where the explicit time dependence stems only from the time dependent tunneling matrix elements.

Let us now turn to the derivation of the Hamiltonian (4) and (5). The microscopic starting point is

$$
H(t)=H_{D}(t)+H_{R}(t)+H_{B}+V_{D B}+H_{T}(t),
$$

where $H_{D}(t), H_{R}(t)$ and $H_{B}$ denote the Hamiltonians for the dot, the reservoirs and the heat bath, respectively. $V_{D B}$ describes the interaction between dot and heat bath and $H_{T}(t)$ the tunneling between dot and reservoirs.

The general form of the dot Hamiltonian is $H_{D}(t)=H_{D}^{0}+e V_{e x}(t)$, where $e V_{e x}(t)$ is the potential energy from the fixed voltage distribution on the reservoirs whereas the electrostatic energy for zero voltage on all reservoirs is included in $H_{D}^{0}$. Screening effects and nearby gates often justify the form $V_{e x}(t)=V_{D}(t) \hat{N}$, where $V_{D}(t)$ is the spatial average of the external electrostatic potential taken over the dot. We denote the normalized and orthogonal many-body eigenfunctions of $H_{D}^{0}$ by $|s\rangle$ with energy $E_{s}$ and obtain $H_{D}(t)=\sum_{s} E_{s} \hat{P}_{s}+e V_{D}(t) \hat{N}$. 
For the reservoir Hamiltonian $H_{R}(t)$ we take a noninteracting Fermi liquid with perfect screening properties like in an ideal metal $H_{R}(t)=$ $\sum_{k r} \epsilon_{k}^{r} a_{k r}^{\dagger} a_{k r}+e \sum_{r} V_{r}(t) \hat{N}_{r}$, where $V_{r}(t)$ is the electrostatic potential of reservoir $r$, and $\hat{N}_{r}$ is the particle number operator.

Tunneling between reservoirs and island is described by

$$
H_{T}(t)=\sum_{r, k l} T_{k l}^{r}(t) a_{k r}^{\dagger} a_{l D}+(\text { h.c. })
$$

where $T_{k l}^{r}(t)$ are possibly time-dependent tunneling matrix elements and $a_{l D}$ is a field operator corresponding to a set of single particle states on the dot. The tunneling matrix elements are described by the spectral function ${ }_{l l^{\prime}}^{r}\left(t, t^{\prime} ; \omega\right)=2 \pi \sum_{k} T_{k l}^{r}(t)^{*} T_{k l^{\prime}}^{r}\left(t^{\prime}\right) \delta\left(\omega-\epsilon_{k}^{r}\right)$. In the time-independent case, one often uses the approximation $,{ }_{l l^{\prime}}^{r}(\omega) \approx \delta_{l l^{\prime}},{ }^{r}$. This assumes constant density of states in the reservoirs as well as the neglect of interference phenomena in higher order perturbation theory in , . Expressed in the basis $\mid s>$ we have $H_{T}(t)=\sum_{r, k, s s^{\prime}} T_{k, s s^{\prime}}^{r}(t) a_{k r}^{\dagger} \hat{P}_{s s^{\prime}}+($ h.c. $)$. The transformed tunneling matrix elements $T_{k, s s^{\prime}}^{r}(t)=\sum_{l} T_{k l}^{r}(t)<s\left|a_{l D}\right| s^{\prime}>$ involve matrix elements of the field operator $a_{l D}$ between many-body states of the island. They can lead to exclusion rules [23, 24]. The function, in the basis $|s\rangle$ reads

$$
{ }_{s_{1} s_{1}^{\prime}, s_{2} s_{2}^{\prime}}^{r}\left(t_{1}, t_{2} ; \omega\right)=2 \pi \sum_{k} T_{k, s_{1} s_{1}^{\prime}}^{r}\left(t_{1}\right)^{*} T_{k, s_{2}^{\prime} s_{2}}^{r}\left(t_{2}\right) \delta\left(\omega-\epsilon_{k}^{r}\right) .
$$

The heat bath $H_{B}$ is modelled by a set of harmonic oscillators $H_{B}=$ $\sum_{q} \omega_{q} b_{q}^{\dagger} b_{q}$ which couple to the particle number operator $\hat{N}$ of the island by the interaction term

$$
V_{D B}=\hat{N} \sum_{q} g_{q}\left(b_{q}+b_{q}^{\dagger}\right)+\hat{N}^{2} \sum_{q} \frac{g_{q}^{2}}{\omega_{q}} .
$$

The second term is a counter-term which is necessary to avoid an unphysical renormalization of the energies $E_{s}$ (see below). The first term describes a fluctuating electrochemical potential on the island.

Finally we perform a standard time-dependent unitary transformation $U(t)$ to bring the Hamiltonian into the most convenient form. We choose $U(t)=\exp \left\{i e \int_{t_{0}}^{t} d t^{\prime}\left(\sum_{r} V_{r}\left(t^{\prime}\right) \hat{N}_{r}+V_{D}\left(t^{\prime}\right) \hat{N}\right)\right\} \exp (i \hat{N} \hat{\phi})$, where $t_{0}$ is the initial time and the hermitian bosonic field $\hat{\phi}$ is defined by $\hat{\phi}=i \sum_{q} \frac{g_{q}}{\omega_{q}}\left(b_{q}-\right.$ $\left.b_{q}^{\dagger}\right)$. The transformed Hamiltonian $\bar{H}=U H U^{\dagger}+i\left(\frac{d}{d t} U\right) U^{\dagger}$ reads $\bar{H}(t)=$ $\bar{H}_{0}+\bar{H}_{T}(t)$ where $\bar{H}_{0}$ and $\bar{H}_{T}(t)$ are given by (4) and (5), respectively. We see that the last term of (13) has been cancelled. Furthermore, we define 
the coupling function ${ }_{s_{1} s_{1}^{\prime}, s_{2} s_{2}^{\prime}}^{-r}\left(t_{1}, t_{2} ; \omega\right)$ after the unitary transformation by replacing $T$ by $\bar{T}$ in Eq. (12).

For convenience we drop finally the bar on all operators and imply that the Hamiltonians and all observables $A(t) \equiv \bar{A}(t)=U(t) A(t) U(t)^{\dagger}$ are the transformed ones after the unitary transformation. The states $|s\rangle$ together with the projectors $\hat{P}_{s s^{\prime}}$ are kept unchanged.

\subsubsection{Metallic island}

A metallic island is characterized by a very small level spacing $\delta E \ll T$ which means that a very large number of excitation energies are relevant. Therefore, following the standard approach, we introduce two approximations from the very beginning. First, like the reservoirs, we treat the island as a Fermi liquid with perfect screening. This means that we use the Coulomb blockade model (2) for the dot Hamiltonian

$$
H_{D}(t)=\sum_{k} \epsilon_{k D} a_{k D}^{\dagger} a_{k D}+E_{C} \hat{N}^{2}+e V_{D}(t) \hat{N}
$$

with $e V_{D}(t)=-2 E_{C} n_{x}(t)$. The total Hamiltonian is again of the form (10) with all other parts given as in the previous section. Secondly, we separate the charge degrees of freedom of the island (described by $N$ ) from the degrees of freedom describing how the particles on the island are distributed among the single particle states (described by $n_{k}^{D}$ ). Furthermore we fix the distribution function on the island by a Fermi distribution. These approximations are justified since the time scale for the change of the distribution function is much larger than the time scale for the variation of the total particle number. To formulate this more precisely we enlarge first our Hilbert space by introducing formal charge states $|N\rangle$ with $N$ ranging from plus to minus infinity. We define the operator $\hat{N}$ in (14) by $\hat{N}|N>=N| N>$ and the projectors $\hat{P}_{N N^{\prime}}=|N\rangle<N^{\prime} \mid$. We demand that each time an electron changes its position from some reservoir to the island or vice versa via tunneling, the charge state has to change simultaneously from $|N\rangle$ to $|N \pm 1\rangle$. This is achieved by introducing the projectors $\hat{P}_{N \pm 1, N}$ into the tunneling Hamiltonian $H_{T}(t)=\sum_{r, k l, N} T_{k l}^{r}(t) a_{k r}^{\dagger} a_{l D} \hat{P}_{N-1, N}+$ (h.c.). By construction, the new Hamiltonian is exactly equivalent to the old one provided we use the constraint $N=\sum_{k} n_{k}^{D}-N_{0}$ to restrict ourselves to the original physical Hilbert space. The approximation formulated above corresponds to the neglect of the latter constraint. Although the corrections have never been analysed systematically it appears reasonable that they are finite-size corrections and scale like the inverse volume of the island.

We now apply the same unitary transformation $U(t)$ as in the previous section. The result for the transformed Hamiltonian is $\bar{H}(t)=\bar{H}_{0}+\bar{H}_{T}(t)$ 
with

$$
\begin{aligned}
\bar{H}_{0} & =\bar{H}_{R}+\bar{H}_{B}+\bar{H}_{C} \\
& =\sum_{r=L, R, D} \sum_{k} \epsilon_{k}^{r} a_{k r}^{\dagger} a_{k r}+\sum_{q} \omega_{q} b_{q}^{\dagger} b_{q}+\sum_{N} E_{N} \hat{P}_{N}, \\
\bar{H}_{T}(t) & \left.=\sum_{r=L, R} \sum_{k l, N} \bar{T}_{k l}^{r}(t) a_{k r}^{\dagger} a_{l D} \hat{P}_{N-1, N} e^{-i \hat{\phi}}+\text { (h.c. }\right),
\end{aligned}
$$

where $\bar{T}_{k l}^{r}(t)$ is defined analog to $(6)$. As indicated we decomposed the dot Hamiltonian (14) into a "reservoir" part $\sum_{k} \epsilon_{k D} a_{k D}^{\dagger} a_{k D}$, which has been included in $\bar{H}_{R}$, and a charge part $\bar{H}_{C}=\sum_{N} E_{N} \hat{P}_{N}$, with $E_{N}=E_{C} N^{2}$.

Following the previous section, we drop from now on all bars and identify $A \equiv \bar{A}=U A U^{\dagger}$ for all observables $A$. Furthermore, using the analog derivation to the quantum dot case, we obtain for the tunneling current operator

$$
\hat{I}_{r}^{t u n}(t)=i e \sum_{k l, N} \bar{T}_{k l}^{r}(t) a_{k r}^{\dagger} a_{l D} \hat{P}_{N-1, N} e^{-i \hat{\phi}}+(\text { h.c. }),
$$

whereas the displacement current can be calculated from (8).

\section{Real-time transport theory}

\subsection{GENERAL CONCEPT}

In this section we will explain the general structure of the theory without going into details of technical derivations. The full microscopic approach together with explicit expressions for various quantities introduced here will be presented in the next section 3.2.

The following considerations refer to the quantum dot case but hold as well for the metallic island by the replacement of dot states by charge states (formally $D \rightarrow C, s \rightarrow N$ ).

The tunneling part $H_{T}(t)$ describes the coupling between the environment (reservoirs and heat bath) and the dot. It will drive the dot system out of equilibrium. For $t \leq t_{0}$, we assume $H_{T}(t)$ to vanish, and the environment to be in equilibrium. This means that the initial density matrix can be written in factorized form $\rho\left(t_{0}\right)=\rho_{R}^{e q} \rho_{B}^{e q} \hat{P}\left(t_{0}\right)$, where $\rho_{R}^{e q} \rho_{B}^{e q}$ is the grandcanonical equilibrium density matrix of the environment, and $\hat{P}(t)$ is the reduced density matrix of the $\operatorname{dot} \hat{P}(t)=\operatorname{Tr}_{R B} \rho(t)$ with $\operatorname{Tr}_{R B}=\operatorname{Tr}_{R} \operatorname{Tr}_{B}$ being the trace over the reservoir and heat bath degrees of freedom.

At time $t_{0}$ we switch on the tunneling between dot and reservoirs. For $t_{0} \rightarrow-\infty$ this is performed adiabatically. Our first aim is to study the time evolution of $\hat{P}(t)$. This will be performed in section 3.2 by integrating 
out the reservoirs and the heat bath with the result of an effective theory in terms of the dot degrees of freedom. We will obtain the formally exact kinetic equation

$$
\frac{d}{d t} P_{s s^{\prime}}(t)+i\left(E_{s}-E_{s^{\prime}}\right) P_{s s^{\prime}}(t)=\sum_{s_{1} s_{1}^{\prime}} \int_{t_{0}}^{t} d t^{\prime} \Sigma\left(t, t^{\prime}\right)_{s s^{\prime}, s_{1} s_{1}^{\prime}} P\left(t^{\prime}\right)_{s_{1} s_{1}^{\prime}},
$$

where $P_{s s^{\prime}}(t)=\left\langle s|\hat{P}(t)| s^{\prime}>\right.$. The second term on the 1.h.s. of this equation is a flow term which describes the time evolution of the density matrix in the absence of tunneling. This is not a dissipative source and, in the absence of tunneling, would lead to a coherent time evolution of the dot. Dissipation is described by the r.h.s. of Eq. (18). It forces the dot to approach a stationary state and is due to tunneling.

The kinetic equation (18) can be written in a more familiar and transparent form by eliminating the nondiagonal matrix elements of the probability distribution. This leads to

$$
\frac{d}{d t} P_{s}(t)=\sum_{s^{\prime}} \int_{t_{0}}^{t} d t^{\prime} \Sigma_{s s^{\prime}}\left(t, t^{\prime}\right) P_{s^{\prime}}\left(t^{\prime}\right) .
$$

Using the property $\sum_{s} \Sigma_{s s^{\prime}}\left(t, t^{\prime}\right)=0$, which is proven in section 3.2 and guarantees the conservation of probability $\sum_{s} \dot{P}_{s}(t)=0$, we can rewrite the kinetic equation as

$$
\frac{d}{d t} P_{s}(t)=\sum_{s^{\prime} \neq s} \int_{t_{0}}^{t} d t^{\prime}\left\{\Sigma_{s s^{\prime}}\left(t, t^{\prime}\right) P_{s^{\prime}}\left(t^{\prime}\right)-\Sigma_{s^{\prime} s}\left(t, t^{\prime}\right) P_{s}\left(t^{\prime}\right)\right\} .
$$

We have obtained the structure of a master equation with a gain and loss term on the r.h.s.. The kernel $\Sigma_{s s^{\prime}}\left(t, t^{\prime}\right)$ can be interpreted as a generalized and formally exact transition rate from the state $s^{\prime}$ at time $t^{\prime}$ to the state $s$ at time $t$. In second order in $H_{T}$, we obtain the lowest order expression for the rate but for arbitrary time-dependent situations. In the asymptotic limit $t_{0} \rightarrow-\infty$ it reduces to the golden rule rate when integrated over the time difference $t-t^{\prime}$ (see at the end of this section and section 4.1). In the context of Coulomb blockade phenomena, this term is called the transition rate of "sequential tunneling". It corresponds to the physical situation where all tunneling processes are incoherent. The next term, which is of forth order in $H_{T}$, is called the cotunneling transition rate. It means that at least two tunneling processes are correlated allowing for coherent transport through the dot from one reservoir to the other. The higher order terms contain processes where the electron tunnels coherently back and forth between the dot and the reservoirs. We will see in section 4 that this can lead to renormalization and broadening effects. Except for special 
systems which are exactly solvable (see section 4.2 for an example), it is not possible to calculate $\Sigma$ exactly. However, we will at least formulate a systematic and very general approximation in section 3.2 which will be applied to specific examples in section 4 . We call the kernel $\Sigma_{s s^{\prime}}\left(t, t^{\prime}\right)$ within this approximation the resonant tunneling transition rate.

For the special case of a diagonal density matrix $P_{s s^{\prime}}(t)=\delta_{s s^{\prime}} P_{s}(t)$, the kernel is given by $\Sigma_{s s^{\prime}}\left(t, t^{\prime}\right)=\Sigma_{s s, s^{\prime} s^{\prime}}\left(t, t^{\prime}\right)$. As we will see in section 4 , there are special systems with the property that $\hat{P}(t)$ will be diagonal for all times $t$ if it is diagonal at the initial time $t_{0}$. To give a concrete example we note the following property of $P_{s s^{\prime}}(t)$ which follows from particle number conservation $P_{s s^{\prime}}(t) \sim \delta_{N_{s}, N_{s^{\prime}}}$, which is fulfilled for all times if it is fulfilled initially. Here, $N_{s}=\langle s|\hat{N}| s>$ denotes the particle number on the dot for state $|s\rangle$. This follows directly from the definition $P_{s s^{\prime}}(t)=<s\left|\operatorname{Tr}_{R B} \rho(t)\right| s^{\prime}>$ and the fact that the total particle number $N_{t o t}=\sum_{r=L, R} N_{r}+N$ is a conserved quantity. Thus, for metallic systems, we find $P_{N N^{\prime}}(t)=\delta_{N N^{\prime}} P_{N}(t)$ if this property holds initially. A similiar proof can also be given for quantum dots with a single spin $1 / 2$ state where spin conservation can be used (see section 4.3).

Another quantity of interest is the tunneling current given by the average of the corresponding operator $I_{r}^{\text {tun }}(t)=\operatorname{Tr} \rho(t) \hat{I}_{r}^{t u n}(t)$. Inserting the form (9) or (17) for the operator and again integrating out the reservoir and heat bath degrees of freedom, we will show in section 3.2 that the tunneling current can be written as

$$
I_{r}^{\text {tun }}(t)=-e \sum_{s s^{\prime}} \int_{t_{0}}^{t} d t^{\prime} \Sigma_{s s^{\prime}}^{r}\left(t, t^{\prime}\right) P_{s^{\prime}}\left(t^{\prime}\right),
$$

where we have already eliminated the nondiagonal elements.

The physical interpretation of $(21)$ is very obvious. To obtain the tunneling current at time $t$, one has to multiply the current rate $\sum_{s} \sum_{s s^{\prime}}^{r}\left(t, t^{\prime}\right)$, corresponding to the sum over all processes starting at $t^{\prime}$ in state $s^{\prime}$ and ending at time $t$ in any state, with the appropriate initial probability $P_{s^{\prime}}\left(t^{\prime}\right)$ and finally integrate over all initial times $t^{\prime}$ and sum over all initial states $\left|s^{\prime}\right\rangle$. The index $r$ indicates that during these processes the particle number in reservoir $r$ has changed. As a minor remark we note that, just for formal reasons, only the sum over $s$ of $\Sigma_{s s^{\prime}}^{r}\left(t, t^{\prime}\right)$ is allowed to be interpreted as the current rate.

The current rate includes all possible processes, i.e. the change of the particle number in reservoir $r$ can take any value. Therefore it is natural to decompose the current rate in the form

$$
\sum_{s} \Sigma_{s s^{\prime}}^{r}\left(t, t^{\prime}\right)=-\sum_{s} \sum_{p=-\infty}^{\infty} p \Sigma_{s s^{\prime}}^{r p}\left(t, t^{\prime}\right),
$$


where $\Sigma_{s s^{\prime}}^{r p}\left(t, t^{\prime}\right)$ corresponds to that part of the total transition rate $\Sigma_{s s^{\prime}}\left(t, t^{\prime}\right)$ where in sum $p$ particles are taken out of reservoir $r$. This allows a decomposition of the tunneling current into a tunneling "in" and a tunneling "out" contribution

$$
I_{r}^{t u n}(t)=e \sum_{p=1}^{\infty} p \sum_{s s^{\prime}} \int_{t_{0}}^{t} d t^{\prime}\left\{\Sigma_{s s^{\prime}}^{r, p}\left(t, t^{\prime}\right) P_{s^{\prime}}\left(t^{\prime}\right)-\Sigma_{s s^{\prime}}^{r,-p}\left(t, t^{\prime}\right) P_{s^{\prime}}\left(t^{\prime}\right)\right\} .
$$

Finally we show how the kinetic equation and the tunneling current can be written in Fourier space. We are interested in the stationary solution and set the initial time $t_{0}=-\infty$. We assume that the time-dependence of the voltages and the tunneling matrix elements is periodic in time with period $T=2 \pi / \Omega$. This implies $\Sigma_{s s^{\prime}}\left(t+T, t^{\prime}+T\right)=\Sigma_{s s^{\prime}}\left(t, t^{\prime}\right)$ and the periodicity of the stationary probability distribution and the tunneling current. Thus, we use the Fourier expansion $P_{s}(t)=\sum_{n=-\infty}^{\infty} P_{s}^{n} e^{i n \Omega t}$, $I_{r}(t)=\sum_{n=-\infty}^{\infty} I_{r}^{n} e^{i n \Omega t}, \Sigma_{s s^{\prime}}\left(t, t^{\prime}\right)=\sum_{n=-\infty}^{\infty} \Sigma_{s s^{\prime}}^{n}\left(t-t^{\prime}\right) e^{i n \Omega t^{\prime}}$, and a corresponding representation for $\Sigma_{s s^{\prime}}^{r}\left(t, t^{\prime}\right)$. Inserting these expansions in the kinetic equation (19) and the tunneling current (21), we find

$$
\begin{aligned}
i n \Omega P_{s}^{n} & =\sum_{s^{\prime}} \sum_{m} \Sigma_{s s^{\prime}}^{m n} P_{s^{\prime}}^{n-m}, \\
I_{r}^{n} & =-e \sum_{s s^{\prime}} \sum_{m} \Sigma_{s s^{\prime}}^{r, m n} P_{s^{\prime}}^{n-m},
\end{aligned}
$$

where we have defined the Laplace transform $\sum_{s s^{\prime}}^{n m}=\int_{0}^{\infty} d \tau \sum_{s s^{\prime}}^{n}(\tau) e^{-i m \Omega \tau}$, and analog for $\Sigma_{s s^{\prime}}^{r, n m}$.

If the period $T$ is much smaller than the characteristic memory time $\tau_{\Sigma}$ of the kernels, the $n=0$ component of $\Sigma_{s s^{\prime}}^{n}\left(t-t^{\prime}\right)$ will give the most dominant contribution to (19). The reason is that, for $n \neq 0$, the factor $e^{i n \Omega t^{\prime}}$ will oscillate very strongly for $t^{\prime}$ varying on a range $\tau_{\Sigma} \gg \Omega^{-1}$. With the same argument one can also neglect the components of $\Sigma_{s s^{\prime}}^{n m}\left(t, t^{\prime}\right)$ for $m \neq 0$. The AC-components of the probability distribution are then much smaller than the DC-components and we obtain

$$
\begin{aligned}
0 & =\sum_{s^{\prime}} \Sigma_{s s^{\prime}} P_{s^{\prime}}=\sum_{s^{\prime} \neq s}\left(\Sigma_{s s^{\prime}} P_{s^{\prime}}-\Sigma_{s^{\prime} s} P_{s}\right), \\
I_{r} & =-e \sum_{s s^{\prime}} \Sigma_{s s^{\prime}}^{r} P_{s^{\prime}},
\end{aligned}
$$

where, by convention, we imply always that we mean the DC-Fourier component $n=m=0$ if no time argument and no Fourier index is written. For time-translational invariant systems, the kernels depend only on the relative time argument $t-t^{\prime}$ and Eqs. (26)-(27) hold exactly. 


$$
\mathrm{P}_{\mathrm{ss}},(\mathrm{t})=\sum_{\overline{\mathrm{s}} \overline{\mathrm{s}}^{\prime}} \mathrm{P}_{\mathrm{s}^{\prime} \overline{\mathrm{s}}}\left(\mathrm{t}_{\mathbf{0}}\right)
$$

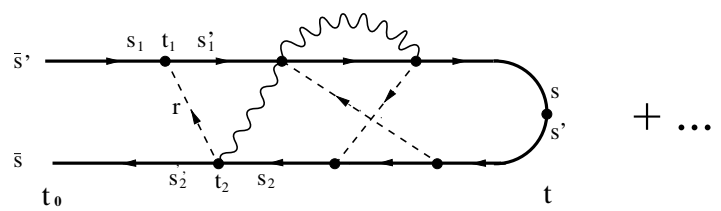

Figure 3. An example for a diagram contributing to the matrix element $P_{s s^{\prime}}(t)$ of the reduced density matrix of the dot. Reservoir (boson) lines are indicated by dashed (wiggly) lines.

\subsection{MICROSCOPIC THEORY}

In this section we provide the microscopic basis for the equations set up in the previous section. We start from the definition $P_{s s^{\prime}}(t)=\operatorname{Tr} \rho(t) \hat{P}_{s^{\prime} s}$ of the matrix elements of the reduced density matrix of the dot and find in interaction picture with respect to $H_{0}=H_{R}+H_{B}+H_{D}$

$$
P_{s s^{\prime}}(t)=\operatorname{Tr} \rho\left(t_{0}\right) T_{\gamma}\left\{e^{-i \int_{\gamma} d t^{\prime} H_{T}\left(t^{\prime}\right)_{I}} \hat{P}_{s^{\prime} s}(t)_{I}\right\}
$$

where $H_{T}(t)_{I}$ and $\hat{P}_{s^{\prime} s}(t)_{I}$ are operators in interaction picture, and $\gamma$ denotes the usual closed Keldysh contour which runs from $t_{0}$ to $t$ on the real axis and then back again from $t$ to $t_{0} . T_{\gamma}$ denotes the time ordering along this closed time path. Using the initial condition for the density matrix and taking matrix elements we obtain

$$
P_{s s^{\prime}}(t)=\sum_{\bar{s} \bar{s}^{\prime}} P_{\bar{s}^{\prime} \bar{s}}\left(t_{0}\right)<\bar{s}\left|\operatorname{Tr}_{R B} \rho_{R}^{e q} \rho_{B}^{e q} T_{\gamma}\left\{e^{-i \int_{\gamma} d t^{\prime} H_{T}\left(t^{\prime}\right)_{I}} \hat{P}_{s^{\prime} s}(t)_{I}\right\}\right| \bar{s}^{\prime}>.
$$

The next step is to expand (29) in $H_{T}(t)_{I}$ and insert the form (5) or (16) for the tunneling Hamiltonian. The tunneling vertices are arranged along the closed time path as indicated in Fig. 3. The upper line corresponds to the forward propagator and the lower line to the backward propagator. To each vertex we assign a time variable $t_{i}$ and, from the tunneling Hamiltonian, a projection operator $\hat{P}_{s_{i}^{\prime} s_{i}}$, where $s_{i}$ is the ingoing state and $s_{i}^{\prime}$ the outgoing state at each vertex (see Fig. 3). There is one external vertex emerging from the projector $\hat{P}_{s^{\prime} s}$ in Eq. (29), which is the rightmost vertex at time $t$ in Fig. 3 . It is the only vertex which does not contain any reservoir or heat bath field operator.

The procedure is now to perform the trace over the reservoirs and the heat bath, and finally calculate the matrix element with respect to the dot states. The trace can be calculated exactly since $H_{0}$ is a bilinear form in the reservoir and boson field operators, and $\rho_{R, B}^{e q}$ are equilibrium density matrices. What is left for each term is a c-number multiplied with the matrix 
element $\left\langle\bar{s}|\ldots| \bar{s}^{\prime}>\right.$ of a product of dot projection operators in interaction picture. We note that the three steps, i.e. calculating $\operatorname{Tr}_{R}, \operatorname{Tr}_{B}$ and the matrix element of the dot operators, can be performed independently since $H_{0}=H_{R}+H_{B}+H_{D}$ contains no coupling between reservoirs, heat bath and dot. Furthermore, the reader can convince himself that Fermi statistics does not give any minus sign during the factorization of reservoir from dot field operators if both are kept in the same sequence separately. This is due to the quadratic structure $a_{k r}^{\dagger} a_{l D}$ or $a_{l D}^{\dagger} a_{k r}$ of the tunneling vertex. In our convention, the time-ordering operator $T_{\gamma}$ does not introduce any change of sign.

Let us start with the calculation of $\operatorname{Tr}_{R}$. It can be performed using Wick's theorem with the result that all reservoir field operators are contracted in pairs of creation and annihilation operators. In our convention, a single contraction for the quantum dot case gives the contribution $( \pm$ refers to $t_{1}>t_{2}$ with respect to the Keldysh time path)

$$
\begin{aligned}
\gamma_{s_{1} s_{1}^{\prime}, s_{2} s_{2}^{\prime}}^{r, \pm}\left(t_{1}, t_{2}\right) & =\sum_{k} \bar{T}_{k, s_{1} s_{1}^{\prime}}^{r}\left(t_{1}\right)^{*} \bar{T}_{k, s_{2}^{\prime} s_{2}}^{r}\left(t_{2}\right)\left\langle T_{\gamma}\left\{a_{k r}\left(t_{1}\right)_{I} a_{k r}^{\dagger}\left(t_{2}\right)_{I}\right\}\right\rangle_{\rho_{R}^{e q}} \\
& =\frac{1}{2 \pi} \int d \omega^{-}{ }_{s_{1} s_{1}^{\prime}, s_{2} s_{2}^{\prime}}\left(t_{1}, t_{2} ; \omega\right) f^{ \pm}(\omega) e^{-i \omega\left(t_{1}-t_{2}\right)}
\end{aligned}
$$

whereas for the metallic case we get

$$
\begin{aligned}
\alpha_{r}^{ \pm}\left(t_{1}, t_{2}\right) & =\sum_{k l} \bar{T}_{k l}^{r}\left(t_{1}\right)^{*} \bar{T}_{k l}^{r}\left(t_{2}\right)\left\langle T_{\gamma}\left\{\left(a_{l D}^{\dagger} a_{k r}\right)\left(t_{1}\right)_{I}\left(a_{k r}^{\dagger} a_{l D}\right)\left(t_{2}\right)_{I}\right\}\right\rangle_{\rho_{R}^{e q}} \\
& =\frac{1}{\pi} \int d \omega \bar{D}_{r}\left(t_{1}, t_{2} ; \omega\right) n^{ \pm}(\omega) e^{-i \omega\left(t_{1}-t_{2}\right)}
\end{aligned}
$$

with $f^{+}=f, f^{-}=1-f, n^{+}=n, n^{-}=1+n$, and $f(\omega), n(\omega)$ being the Fermi and Bose distribution function. Furthermore, we have defined

$$
\frac{1}{\pi} D_{r}\left(t, t^{\prime} ; \omega\right)=\frac{1}{2 \pi} \sum_{l}, \stackrel{r}{l l}\left(t, t^{\prime} ; \omega+\epsilon_{l}^{D}\right)\left[f\left(\epsilon_{l}^{D}\right)-f\left(\epsilon_{l}^{D}+\omega\right)\right],
$$

and $\bar{D}$ by replacing, $\rightarrow$,

For the metallic case we have used the fact that each loop of Wick contractions is proportional to the transverse channel number $Z$. Therefore, for large channel number, the loops will contain the minimal number of vertices, i.e. they have the form of Eq. (31). Experimentally, the channel number is usually of order $10^{3}$.

Eq. (32) can be written in a more explicit form if we assume a constant density of states $\nu_{D}$ in the island, take tunneling matrix elements of the 
form $T_{k l}^{r}(t)=F_{r}(t) T_{k l}^{r}$, and use approximately $,{ }_{l l}^{r}(\omega) \approx,{ }^{r}$ independent of $l$ and $\omega$. We obtain $D_{r}\left(t, t^{\prime} ; \omega\right)=F_{r}(t)^{*} F_{r}\left(t^{\prime}\right) D_{r}(\omega)$ with

$$
\frac{1}{\pi} D_{r}(\omega)=\alpha_{0}^{r} \omega
$$

and $\alpha_{0}^{r}=1 /(2 \pi),{ }^{r} \nu_{D}=R_{K} /\left(4 \pi^{2} R_{T}^{r}\right)$ is proportional to the conductance $G_{T}^{r}=1 / R_{T}^{r}$ of a single barrier connecting the island to reservoir $r=L, R$ in units of the quantum conductance $G_{K}=1 / R_{K}=e^{2} / h$.

For the quantum dot case, we get a minus sign for each crossing of contractions due to Fermi statistics. Diagrammatically, a contraction between reservoir field operators is indicated by a dashed line (see Fig. 3). The direction of the line is chosen in such a way that it leaves the vertex where a particle is annihilated on the dot. The time argument of this vertex has to be chosen as the second time argument of the functions $\gamma$ and $\alpha$, i.e. corresponds to $t_{2}$ in Eq. (30) and (31). The states $s_{1,2}\left(s_{1,2}^{\prime}\right)$ refer to the ingoing (outgoing) dot states at both vertices.

The calculation of $\operatorname{Tr}_{B}$ proceeds in a different way since the tunneling vertex contains an exponential $\exp ( \pm i \hat{\phi})$ of a linear bosonic field. Here we can use path integral methods or Feynman's disentangling method [28] to get

$$
\begin{aligned}
& \left\langle T_{\gamma}\left\{e^{-i \hat{\phi}\left(t_{1}\right)_{I}} e^{i \hat{\phi}\left(t_{1}^{\prime}\right)_{I}} \ldots e^{-i \hat{\phi}\left(t_{m}\right)_{I}} e^{i \hat{\phi}\left(t_{m}^{\prime}\right)_{I}}\right\}\right\rangle_{\rho_{B}^{e q}}= \\
& \quad=\prod_{i<j} P^{ \pm}\left(t_{i}, t_{j}\right)^{-1} \prod_{i<j} P^{ \pm}\left(t_{i}^{\prime}, t_{j}^{\prime}\right)^{-1} \prod_{i, j} P^{ \pm}\left(t_{i}, t_{j}^{\prime}\right),
\end{aligned}
$$

where, for $t_{1}>t_{2}$ with respect to the Keldysh path, we have defined the correlators $P^{ \pm}\left(t_{1}, t_{2}\right)=\left\langle T_{\gamma}\left\{e^{-i \hat{\phi}\left(t_{1}\right)_{I}} e^{i \hat{\phi}\left(t_{2}\right)_{I}}\right\}\right\rangle_{\rho_{B}^{e q}}=P\left( \pm\left(t_{1}-t_{2}\right)\right)$ with $P(t)=e^{-W(-t)}, W(t)=S(t)+i R(t)$, and $[20,27]$

$$
\begin{aligned}
& S(t)=\frac{1}{\pi} \int_{0}^{\infty} d \omega \frac{J(\omega)}{\omega^{2}}(1-\cos (\omega t)) \operatorname{coth}\left(\frac{\beta_{B} \omega}{2}\right), \\
& R(t)=\frac{1}{\pi} \int_{0}^{\infty} d \omega \frac{J(\omega)}{\omega^{2}} \sin (\omega t),
\end{aligned}
$$

where $J(\omega)$ is defined in $(7)$. The Fourier transform $P(E)=\frac{1}{2 \pi} \int d t e^{i E t} P(t)$ describes the probability that an electron absorbs the energy $E$ from the bosonic environment $[26,27]$. We write (34) formally as a sum by defining $L_{d}^{ \pm}\left(t_{1}, t_{2}\right)=P^{ \pm}\left(t_{1}, t_{2}\right)-1$ and $L_{s}^{ \pm}\left(t_{1}, t_{2}\right)=P^{ \pm}\left(t_{1}, t_{2}\right)^{-1}-1$. Here, $L_{d}$ corresponds to a pair of vertices with different signs of the bosonic phase fields, whereas $L_{s}$ refers to a pair with the same sign. Both $L_{d}$ and $L_{s}$ are zero if the coupling to the environment is absent. Diagrammatically, 
we represent the bosonic contributions $L_{d, s}$ by wiggly lines (see Fig. 3 ). In contrast to reservoir lines, an arbitrary number of bosonic lines can be attached to a single vertex.

The matrix element $\left\langle\bar{s}|\ldots| \bar{s}^{\prime}>\right.$ of products of dot projection operators in interaction picture is given by

$$
\left.\left.<\bar{s}\left|\prod_{i=1}^{m} \hat{P}_{s_{i}^{\prime} s_{i}}\left(t_{i}\right)_{I}\right| \bar{s}^{\prime}\right\rangle=\prod_{i=0}^{m}<s_{i+1}\left|U_{D}\left(t_{i+1}, t_{i}\right)\right| s_{i}^{\prime}\right\rangle
$$

where $U_{D}\left(t, t^{\prime}\right)$ is the evolution operator of $H_{D}$, and we identified $s_{m+1}=\bar{s}$, $s_{0}^{\prime}=\bar{s}^{\prime}$, and $t_{m+1}=t_{0}$. This result means that each segment of the Keldysh contour in Fig. 3, which connects two vertices, corresponds to a matrix element of the dot evolution operator starting from the outgoing state of the initial vertex to the incoming state of the final vertex. Since $H_{D}$ is diagonal in the states $|s\rangle$, the matrix element of the evolution operator is given by $\left\langle s\left|U_{D}\left(t, t^{\prime}\right)\right| s^{\prime}\right\rangle=\delta_{s s^{\prime}} e^{-i E_{s}\left(t-t^{\prime}\right)}$. This means that we can assign a certain dot state to each segment of the Keldysh contour. However, for more general dot Hamiltonians, which include transitions between the dot states $\mid s>$, one has to use the above description in terms of the dot evolution operator.

Finally we have to assign the factor $(-i)^{n} i^{m}$ to each diagram which arises from the expansion of the exponentials in Eq. (29). Here, $n(m)$ is the number of vertices on the forward (backward) propagator. The time integrations are then all performed on the real axis from $t_{0}$ to $t$. Assigning a factor $(-i)^{2}$ to each reservoir line, we can alternatively say that each reservoir line and each vertex on the lower line of the Keldysh contour gives rise to a minus sign. Furthermore, each diagram is multiplied with the matrix element $P_{\bar{s}^{\prime}, \bar{s}}\left(t_{0}\right)$ of the initial distribution.

We mention that each reservoir line can be dressed by a bosonic contribution. This means that instead of $\gamma^{r, \pm}$, the contribution of a reservoir line is replaced by $\tilde{\gamma}_{s_{1} s_{1}^{\prime}, s_{2} s_{2}^{\prime}}^{r, \pm}\left(t_{1}, t_{2}\right)=\gamma_{s_{1} s_{1}^{\prime}, s_{2} s_{2}^{\prime}}^{r, \pm}\left(t_{1}, t_{2}\right) P^{ \pm}\left(t_{1}, t_{2}\right)$, where we have added the two contributions of the two vertices being connected by a reservoir and a boson line (giving $\gamma L_{d}$ ), and the term where they are only connected by a reservoir line (giving $\gamma$ ). In the same way we replace $\alpha_{r}^{ \pm}\left(t_{1}, t_{2}\right)$ by $\tilde{\alpha}_{r}^{ \pm}\left(t_{1}, t_{2}\right)=\alpha_{r}^{ \pm}\left(t_{1}, t_{2}\right) P^{ \pm}\left(t_{1}, t_{2}\right)$. In the presence of the heat bath we will automatically imply from now on that a dashed line corresponds to a dressed reservoir line.

The dressing can be written very elegantly in Fourier space if we consider the case of time-independent tunneling matrix elements $T_{k l}^{r}$ and periodic voltages of the harmonic form $e \bar{V}_{r}(t)=e \bar{V}_{r}^{0}+\bar{\Delta}_{r} \sin (\Omega t)$. We define the 


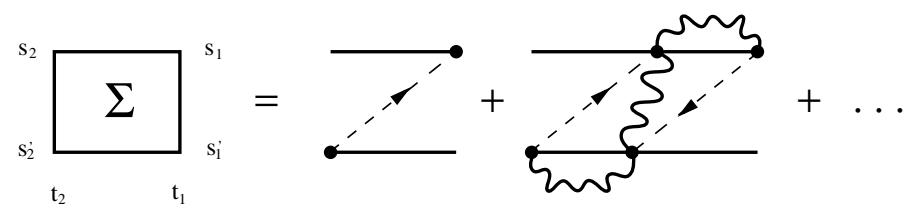

Figure 4. The kernel $\Sigma_{s_{1} s_{1}^{\prime}, s_{2} s_{2}^{\prime}}\left(t_{1}, t_{2}\right)$ which contains all irreducible diagrams in the sense that an arbitrary vertical line will always cut through some reservoir or boson line.

Fourier expansion of $A=\tilde{\gamma}, \tilde{\alpha}$ by

$$
A\left(t_{1}, t_{2}\right)=\sum_{n=-\infty}^{\infty} \int d \omega e^{i n \Omega t_{1}} e^{-i \omega\left(t_{1}-t_{2}\right)} A^{n}(\omega),
$$

which is possible since $A\left(t_{1}+T, t_{2}+T\right)=A\left(t_{1}, t_{2}\right)$. After some algebra we obtain

$$
\begin{aligned}
\tilde{\gamma}_{s_{1} s_{1}^{\prime}, s_{2} s_{2}^{\prime}}^{r, \pm, n}(\omega) & =\frac{1}{2 \pi} \int d \omega^{\prime}{ }_{s_{1} s_{1}^{\prime}, s_{2} s_{2}^{\prime}}^{r}\left(\omega-\omega^{\prime}-e \bar{V}_{r}^{0}\right) f_{r}^{ \pm}\left(\omega-\omega^{\prime}\right) P_{r, n}^{ \pm}\left(\omega^{\prime}\right), \\
\tilde{\alpha}_{r}^{ \pm, n}(\omega) & =\frac{1}{\pi} \int d \omega^{\prime} D_{r}\left(\omega-\omega^{\prime}-\epsilon \bar{V}_{r}^{0}\right) n_{r}^{ \pm}\left(\omega-\omega^{\prime}\right) P_{r, n}^{ \pm}\left(\omega^{\prime}\right)
\end{aligned}
$$

where $f_{r}(\omega)=f\left(\omega-e \bar{V}_{r}^{0}\right), n_{r}(\omega)=n\left(\omega-e \bar{V}_{r}^{0}\right)$, and

$$
P_{r, n}^{ \pm}(\omega)=i^{n} \sum_{m} J_{n+m}\left(\frac{\bar{\Delta}_{r}}{\Omega}\right) J_{m}\left(\frac{\bar{\Delta}_{r}}{\Omega}\right) P^{ \pm}(\omega+m \Omega) .
$$

This shows very clearly the effect of dressing a reservoir line with index $r$ and Fourier component $n$. The heat bath and the time-dependent fields supply the energy $\omega^{\prime}$ for absorption/emission with probability $P_{r, n}^{ \pm}\left(\omega^{\prime}\right)$. Without heat bath we obtain the usual Tien-Gordon theory for the $n=0$ Fourier-component [29].

We can now proceed to derive the kinetic equation (18). Looking at an arbitrary diagram we distinguish between two different time segments. There are "free" time segments in the sense that a vertical line drawn through the diagram will not cut through any reservoir or boson line. These parts correspond to the free evolution of the density matrix of the dot without any coupling to the external environment. All the other time segments are "irreducible", i.e. a vertical line cuts either through a reservoir or a boson line. They reflect the influence of the environment. We denote the sum of all irreducible diagrams by the kernel $\Sigma_{s_{1} s_{1}^{\prime}, s_{2} s_{2}^{\prime}}\left(t_{1}, t_{2}\right)$, with arguments as shown in Fig. 4. The summation of sequences of irreducible blocks with free parts in between can be written in the style of a Dyson equation (see 


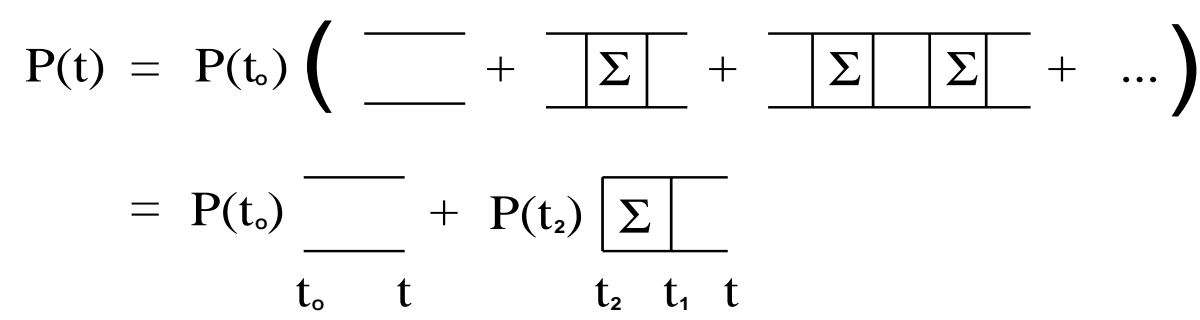

Figure 5. The Dyson-like equation for the probability distribution. $\Sigma$ includes all irreducible diagrams in the sense that any vertical line will at least cut one reservoir or boson line.

Fig. 5)

$$
\begin{aligned}
& P_{s s^{\prime}}(t)=\sum_{\bar{s} \bar{s}^{\prime}} \Pi_{s s^{\prime}, \bar{s} \bar{s}^{\prime}}^{(0)}\left(t, t_{0}\right) P_{\bar{s} \bar{s}^{\prime}}\left(t_{0}\right) \\
& +\sum_{\substack{s_{1} s_{2} \\
s_{1}^{\prime} s_{2}^{\prime}}} \int_{t_{0}}^{t} d t_{1} \int_{t_{0}}^{t_{1}} d t_{2} \Pi_{s s^{\prime}, s_{1} s_{1}^{\prime}}^{(0)}\left(t, t_{1}\right) \Sigma_{s_{1} s_{1}^{\prime}, s_{2} s_{2}^{\prime}}\left(t_{1}, t_{2}\right) P_{s_{2} s_{2}^{\prime}}\left(t_{2}\right),
\end{aligned}
$$

where $\Pi_{s_{1} s_{1}^{\prime}, s_{2} s_{2}^{\prime}}^{(0)}\left(t_{1}, t_{2}\right)=<s_{1}\left|U_{D}\left(t_{1}, t_{2}\right)\right| s_{2}><s_{2}^{\prime}\left|U_{D}\left(t_{2}, t_{1}\right)\right| s_{1}^{\prime}>$ describes the evolution of the density matrix in the free segments. Differentiating (42) with respect to $t$, we arrive at the kinetic equation (18).

For the diagonal kinetic equation (19) we have to define the kernel $\Sigma_{s s^{\prime}}$ in a different way. We allow for free segments in the kernel as well but with the restriction that the dot states associated with the lower and upper line of the contour are different in the free segments.

The property $\sum_{s} \Sigma_{s s^{\prime}}\left(t, t^{\prime}\right)=0$, needed for the derivation of $(20)$, can be easily proven by attaching the rightmost vertex of each diagram $\Sigma$ to the upper and lower proppagator. The minus sign for each vertex on the backward propagator cancels both contributions if we sum over all states $s$.

To calculate the tunneling current, we have to replace the projector $\hat{P}_{s^{\prime} s}$ in (29) by the tunneling current operator (9) (quantum dot case) or (17) (metallic island). This means that the rightmost vertex of each diagram will be the tunneling current vertex which has the same structure as the other tunneling vertices from $H_{T}$. Therefore, the first irreducible block $\Sigma^{r}$ to the right is part of the total kernel $\Sigma$ which enters the kinetic equation. Here $r$ is the index for the reservoir for which we want to calculate the tunneling current. Accounting correctly for the sign of the tunneling current vertex, we find immediately that $\Sigma^{r}$ is that part of $\Sigma$, where the reservoir line attached to the rightmost vertex corresponds to reservoir $r$ and is an outgoing (ingoing) line if the rightmost vertex lies on the upper (lower) 
(a)

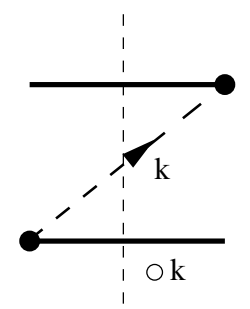

(b)

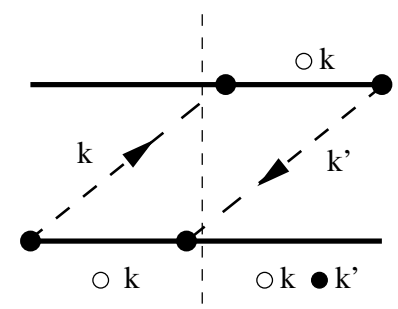

Figure 6. Diagrams contributing to (a) sequential and (b) resonant tunneling. At each reservoir line we have indicated which state $k$ of the reservoir is involved at the tunneling vertices. This creates holes (open circles) or particles (filled circles) on the propagators.

propagator. The other irreducible blocks which follow $\Sigma^{r}$ to the left are identical to $\Sigma$. Thus, after summing over all sequences of $\Sigma$ which gives the probability distribution $P$, we obtain (21). The proof of (22) requires some more technical considerations and can be found in [17].

For a given model it is straightforward to calculate the lowest orders of the kernels $\Sigma$ and $\Sigma^{r}$. However, as we will see in section 4 , renormalization and broadening effects due to quantum fluctuations can only be derived by considering an infinite series of higher order diagrams. We select this series by allowing the total density matrix to be nondiagonal with respect to the reservoir states up to a certain degree. For this let us disregard the bosonic heat bath and consider first the lowest order contribution to the kernels. This is the contribution to the sequential tunneling or golden rule rate and consists diagrammatically of one single reservoir line. An example is shown in Fig.6a. If the reservoir field operator at the tunneling vertices is $a_{k r}^{(\dagger)}$, we see that one hole in reservoir $r$ is present on the backward propagator whereas the forward propagator remains unchanged (we use the states to the left of the diagram as reference). This means that we are considering a matrix element of the total density matrix which is offdiagonal up to one hole excitation. If we consider all diagrams in lowest order, we find that sequential tunneling can be characterized by offdiagonal elements up to one hole or one electron excitation. This shows that the density matrix tries to be as close as possible to a diagonal matrix with respect to the reservoir states. Therefore it is natural to improve sequential tunneling by considering the next possibility of nondiagonal matrix elements, namely those which are offdiagonal up to one electron-hole, electron-electron or hole-hole excitation. An example is shown in Fig.6b. It shows that this approximation can be characterized diagrammatically by the condition that any vertical cut can cut at most two reservoir lines.

Without the heat bath it can be shown that the sum over all diagrams within this approximation can be written in the form of a self-consistent integral equation. For special models this integral equation can be solved 


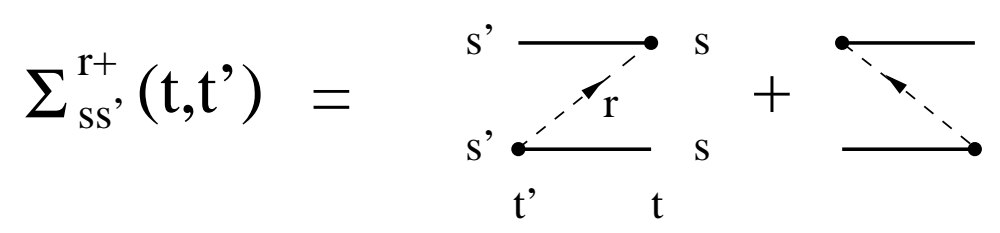

Figure 7. The diagrams for the golden rule rate to tunnel from reservoir $r$ to the dot. The rate to tunnel from the dot to reservoir $r$ is obtained from the same diagrams by inverting the direction of the reservoir lines.

analytically, otherwise one has to find the solution numerically. For the technical details we refer the reader to $[14,17]$. In the presence of a heat bath, one can use the same solution by dressing the reservoir lines. The inclusion of bosonic lines between vertices which are not connected by reservoir lines is very difficult and is still an open problem.

\section{Applications}

In this section we will describe several applications using the formalism we have developed in section 3. We start with two well-known limits which are standardly used in the literature to describe most of the experiments dealing with transport through small devices: golden rule theory (sequential tunneling) and the noninteracting case (Landauer-Büttiker theory). Golden rule theory treats the tunneling in lowest order whereas interaction effects are incorporated in all orders. The noninteracting case disregards interaction effects whereas the tunneling is treated in all orders. In section 4.3 we describe resonant tunneling in a quantum dot with large charging energy and two possible spin excitations, and in section 4.4 resonant tunneling for the infinite- $\mathrm{Z}$ metallic island in the two state approximation. Here tunneling is considered in all orders within the resonant tunneling approximation set up in section 3 , and interaction effects are taken fully into account.

\subsection{SEQUENTIAL TUNNELING}

The rates $\Sigma_{s s^{\prime}}^{r p}\left(t, t^{\prime}\right)$ in second order in $H_{T}$ are shown in Fig. 7 for $s \neq s^{\prime}$. In the current formula (23) we need in lowest order only $\Sigma_{s s^{\prime}}^{r \pm} \equiv \Sigma_{s s^{\prime}}^{r, \pm 1}$ with $s \neq s^{\prime}$. Furthermore, for $s \neq s^{\prime}$, we get for the kernels entering the kinetic equation (20) $\Sigma_{s s^{\prime}}\left(t, t^{\prime}\right)=\sum_{r} \sum_{p= \pm 1} \Sigma_{s s^{\prime}}^{r p}\left(t, t^{\prime}\right)$.

The diagrammatic rules give

$$
\begin{aligned}
& \Sigma_{s s^{\prime}}^{r+}\left(t, t^{\prime}\right)=e^{i\left(E_{s}-E_{s^{\prime}}\right)\left(t-t^{\prime}\right)} \tilde{\gamma}_{s s^{\prime}, s^{\prime} s}^{r+}\left(t, t^{\prime}\right)+\left(t \leftrightarrow t^{\prime}\right), \\
& \Sigma_{s s^{\prime}}^{r-}\left(t, t^{\prime}\right)=e^{-i\left(E_{s}-E_{s^{\prime}}\right)\left(t-t^{\prime}\right)} \tilde{\gamma}_{s^{\prime} s, s s^{\prime}}^{r-}\left(t, t^{\prime}\right)+\left(t \leftrightarrow t^{\prime}\right),
\end{aligned}
$$


for the quantum dot case, and

$$
\Sigma_{N N^{\prime}}^{r \pm}\left(t, t^{\prime}\right)=e^{ \pm i\left(E_{N}-E_{N^{\prime}}\right)\left(t-t^{\prime}\right)} \tilde{\alpha}_{r}^{ \pm}\left(t, t^{\prime}\right) \delta_{N, N^{\prime} \pm 1}+\left(t \leftrightarrow t^{\prime}\right)
$$

for the metallic case with $E_{N}=E_{C} N^{2}$.

Using these results one can, in principle, calculate the full time dependent solution starting from an arbitrary initial state. For the stationary state one needs only the quantities $\Sigma_{s s^{\prime}}^{r p, n m}$ used in (24) and (25). From (43) and the Fourier expansion (38) of $\tilde{\gamma}$, we find for periodic voltages

$$
\begin{aligned}
& \sum_{s s^{\prime}}^{r+, n m}=i \int d \omega \tilde{\gamma}_{s s^{\prime}, s^{\prime} s}^{r,+, n}(\omega) \\
& \left\{\frac{1}{E_{s}-E_{s^{\prime}}-\omega+(n-m) \Omega+i \eta}-\frac{1}{E_{s}-E_{s^{\prime}}-\omega+m \Omega-i \eta}\right\},
\end{aligned}
$$

and a corresponding equation for $\Sigma_{s s^{\prime}}^{r-, n m}$ if we interchange $E_{s} \leftrightarrow E_{s}^{\prime}$ and $\tilde{\gamma}_{s s^{\prime}, s^{\prime} s}^{r,+, n} \leftrightarrow \tilde{\gamma}_{s^{\prime} s, s s^{\prime}}^{r,-, n}$. The metallic case follows from $\tilde{\gamma}_{\ldots, \pm, n}^{r, \pm} \leftrightarrow \tilde{\alpha}_{r}^{ \pm, n} \delta_{N, N^{\prime} \pm 1}$. Without heat bath and using the Coulomb blockade model for the quantum dot, the stationary current has been calculated from these rates in Ref. [35].

Let us discuss some limiting cases which are usually treated in the literature. As discussed in section 3, we can restrict ourselves to the DCcomponents $\Sigma_{s s^{\prime}}^{r \pm} \equiv \Sigma_{s s^{\prime}}^{r \pm, 00}$ if the time-dependent fields are absent or have a very high frequency $\Omega \gg$, . From (46) we get

$$
\sum_{s s^{\prime}}^{r+}=2 \pi \tilde{\gamma}_{s s^{\prime}, s^{\prime} s}^{r+}\left(E_{s}-E_{s^{\prime}}\right) \quad, \quad \sum_{s s^{\prime}}^{r-}=2 \pi \tilde{\gamma}_{s^{\prime} s, s s^{\prime}}^{r-}\left(E_{s^{\prime}}-E_{s}\right) .
$$

and analog for the metallic case by $\tilde{\gamma}_{\ldots, \ldots}^{r, \pm} \leftrightarrow \tilde{\alpha}_{r}^{ \pm, n} \delta_{N, N^{\prime} \pm 1}$.

In the absence of time-dependent fields and the heat bath this gives for the quantum dot case

$$
\begin{aligned}
& \Sigma_{s s^{\prime}}^{r+}={ }^{r} f_{r}^{+}\left(E_{s}-E_{s^{\prime}}\right) \sum_{l}|<s| a_{l D}^{\dagger}\left|s^{\prime}>\right|^{2}, \\
& \Sigma_{s s^{\prime}}^{r-}={ }^{r} f_{r}^{-}\left(E_{s^{\prime}}-E_{s}\right) \sum_{l}|<s| a_{l D}\left|s^{\prime}>\right|^{2},
\end{aligned}
$$

where we have used $(39)$ together with $,{ }_{l l^{\prime}}^{r}(\omega)=\delta_{l l^{\prime}},{ }^{r}$. For metallic islands we get

$$
\begin{aligned}
& \Sigma_{N N^{\prime}}^{r+}=2 \pi \alpha_{0}^{r} \cdot\left(E_{N}-E_{N-1}-e \bar{V}_{r}\right) n_{r}^{+}\left(E_{N}-E_{N-1}\right) \delta_{N, N^{\prime}+1} \\
& \Sigma_{N N^{\prime}}^{r-}=2 \pi \alpha_{0}^{r} \cdot\left(E_{N+1}-E_{N}-e \bar{V}_{r}\right) n_{r}^{-}\left(E_{N+1}-E_{N}\right) \delta_{N, N^{\prime}-1}
\end{aligned}
$$

where we used (40) together with (33).

Eqs. (48)-(51) are the usual golden rule rates which are standardly used in the literature. In the presence of time-dependent voltages and the heat 
bath they have to be convoluted with the probability function $P_{r}^{ \pm} \equiv P_{r, 0}^{ \pm}$ defined in (41). Physically, they express what we have already discussed qualitatively in section 2.1. For a current to flow through the dot we need that both the tunneling "in" and tunneling "out" rates are present. For this let us consider a transition between two dot states $s_{N} \leftrightarrow s_{N+1}$, where $s_{N}$ corresponds to a state with $N$ particles on the dot. For tunneling "in" we have $s^{\prime}=s_{N}$ and $s=s_{N+1}$ in (48). This means that $E_{s_{N+1}}-E_{s_{N}}<e \bar{V}_{r}$ according to the Fermi function in (48). For tunneling "out" we have $s^{\prime}=$ $s_{N+1}$ and $s=s_{N}$ in (49). This gives $E_{s_{N+1}}-E_{s_{N}}>e \bar{V}_{r^{\prime}}$. Both conditions can only be fulfilled simultaneously if the excitation energy $E_{s_{N+1}}-E_{s_{N}}$ lies in the window of two different effective potentials $e \bar{V}_{r^{\prime}}<E_{s_{N+1}}-E_{s_{N}}<e \bar{V}_{r}$. This expresses energy conservation from golden rule and the Pauli principle as already explained in section 2.1. and illustrated in Fig. 2.

The master equation with golden rule rates has been studied extensively in the literature. We mention Ref. [1] for the metallic case, Refs. [30-32] for the Coulomb blockade model, Ref. [23, 33] for the quantum dot case with exact many-body wave functions in the few electron limit, Ref. [34] for the metallic case in time-dependent fields, Ref. [35] for the Coulomb blockade model in time-dependent fields, and Refs. [26, 27] for the metallic case in the presence of a heat bath.

Finally we want to mention that the kernels in lowest order perturbation theory remain the same for the metallic case even if the channel number $Z$ is finite, since the lowest order can contain only one loop with two tunneling vertices.

\section{2. "NONINTERACTING" QUANTUM DOT}

In this section we consider the special case of a quantum dot consisting of one single-particle state in the absence of time-dependent fields and the heat bath. This means that we consider the Hamiltonian

$$
H(t)=\sum_{k r} \epsilon_{k}^{r} a_{k r}^{\dagger} a_{k r}+\epsilon c^{\dagger} c+\sum_{k r}\left(\bar{T}_{k}^{r}(t) a_{k r}^{\dagger} c+h . c .\right),
$$

where $c, c^{\dagger}$ are the field operators of the dot, and the time dependence of the tunneling matrix elements involves only the static effective potentials of the reservoirs $\bar{T}_{k}^{r}(t)=T_{k}^{r} \exp \left(i e \bar{V}_{r}\left(t-t_{0}\right)\right)$. Obviously the Hamiltonian has the form of a noninteracting system which can be solved exactly. Only the presence of the effective potential $\bar{V}_{r}=V_{r}-V_{D}$ within the tunneling matrix elements reminds of the Coulomb interaction. Here, the latter has only the effect of shifting the band buttom of the reservoirs and the dot.

The above Hamiltonian can be thought of as a special case of our general Hamiltonian set up in section 2.2.1. To show this let us consider the case 
where only one excitation energy $E_{s_{1}}-E_{s_{0}}$ of the quantum dot is relevant, with $\mid s_{0}>$ and $\mid s_{1}>$ being two ground states of the dot corresponding to particle numbers $N$ and $N+1$, respectively (e.g. $N=0$ ). In this case, the Hamiltonian from (4) and (5) reads

$$
H(t)=\sum_{k r} \epsilon_{k}^{r} a_{k r}^{\dagger} a_{k r}+\left(E_{s_{1}}-E_{s_{0}}\right) \hat{P}_{s_{1}}+\sum_{k r}\left(\bar{T}_{k, s_{0} s_{1}}^{r}(t) a_{k r}^{\dagger} \hat{P}_{s_{0} s_{1}}+\text { h.c. }\right),
$$

where we have already used $\hat{P}_{s_{0}}+\hat{P}_{s_{1}}=1$ and omitted an overall constant. This form is equivalent to the above Hamiltonian since we can identify $\left.\hat{P}_{s_{0} s_{1}}=c,\left|s_{0}>=\right| 0\right\rangle,\left|s_{1}\right\rangle=\mid 1>, T_{k, s_{0} s_{1}}^{r}=T_{k}^{r}$, and $\epsilon=E_{s_{1}}-E_{s_{0}}$. This means that there is a well-defined limit where an interacting quantum dot can effectively be described by a noninteracting Hamiltonian [36]. However, in a realistic situation degeneracies of excitations can hardly be excluded due to spin and orbital effects, at least in the absence of high magnetic fields. It is only this case where interaction effects really become important and will change the qualitative behaviour of the noninteracting case completely in the whole temperature regime (see section 4.3 ).

The nonequilibrium problem corresponding to the Hamiltonian (52) has been solved exactly by many authors. We mention the Landauer-Büttiker formalism [7, 22, 37], Keldysh technique [36, 38], equation of motion methods [31], and golden rule theory with lorentzian broadening of the energy conservation [35]. Here we will rederive the solution by using the resonant tunneling approximation which turns out to be exact in the noninteracting limit. We only show the analytical result here. For the technical details the reader is refered to Refs. [17].

We first introduce the quantities $\gamma_{r}^{ \pm}(\omega)=\gamma_{01,10}^{r, \pm}(\omega)$ which are, up to a factor $2 \pi$, identical to the golden rule tunneling "in" and "out" rates given by (47) if we set $\omega=\epsilon$. With (39) we obtain $\gamma_{r}^{ \pm}(\omega)=(1 /(2 \pi)),{ }_{r}(\omega) f_{r}^{ \pm}(\omega)$, where we defined,${ }_{r}(\omega)={ }_{01,10}^{r}\left(\omega-e \bar{V}_{r}\right)$

The transition rates within the resonant tunneling approximation are given by

$$
\Sigma_{10}=\frac{1}{\lambda} \int d \omega \frac{\sum_{r},{ }_{r}(\omega) f_{r}^{+}(\omega)}{|\omega-\epsilon-\sigma(\omega)|^{2}} \quad, \quad \Sigma_{01}=\frac{1}{\lambda} \int d \omega \frac{\sum_{r},{ }_{r}(\omega) f_{r}^{-}(\omega)}{|\omega-\epsilon-\sigma(\omega)|^{2}},
$$

where

$$
\sigma(\omega)=\int d \omega^{\prime} \frac{\sum_{r} \gamma_{r}\left(\omega^{\prime}\right)}{\omega-\omega^{\prime}+i \eta}
$$

$\gamma_{r}(\omega)=\gamma_{r}^{+}(\omega)+\gamma_{r}^{-}(\omega)=(1 /(2 \pi)),{ }_{r}(\omega)$, and $\lambda=\int d \omega|\omega-\epsilon-\sigma(\omega)|^{-2}$.

In the numerator of $\mathrm{Eq}$. (54) we recognize the golden rule transition rates. The denominator describes a renormalization and a broadening of 
the dot excitation energy $\epsilon$ by the real and imaginary part of $\sigma(\omega)$. We get

$$
\operatorname{Re} \sigma(\omega)=\frac{1}{2 \pi} P \int d \omega^{\prime} \frac{,\left(\omega^{\prime}\right)}{\omega-\omega^{\prime}} \quad, \quad \operatorname{Im} \sigma(\omega)=-\frac{1}{2},(\omega),
$$

where, $=\sum_{r},{ }_{r}$ and $P \int$ denotes a principal value integral. The renormalization and the broadening are independent of temperature and bias voltage. This is the reason why quantum fluctuations in noninteracting systems do not result in anomalies in the zero-temperature limit. Furthermore, for nearly constant density of states in the reservoirs the energy dependence of ,$(\omega)$ is weak. This results in a small renormalization and a nearly constant broadening.

If , is energy independent we have $\sigma=i, / 2$ and $\lambda=2 \pi /$,. We obtain $\Sigma_{10}=\int d \omega \sum_{r},{ }_{r} f_{r}^{+}(\omega) \delta_{\Gamma}(\omega-\epsilon)$ and $\Sigma_{01}=\int d \omega \sum_{r},{ }_{r} f_{r}^{-}(\omega) \delta_{\Gamma}(\omega-\epsilon)$ for the transition rates. Here, the function $\delta_{\Gamma}(\omega)=(, /(2 \pi)) /\left(\omega^{2}+(, / 2)^{2}\right)^{-1}$ has a lorentzian form with half-width, . If we replace this function by a Dirac delta function we would obtain the golden rule theory. This result expresses a very important feature of noninteracting systems with constant , . One can just use elementary golden rule theory and obtains the exact solution by smearing out the energy conservation by,$!$ It is remarkable that this property even holds when time-dependent fields are present [35]. It is basically due to the fact that the broadening of the dot excitation energy is a constant and does not depend on energy, temperature or bias voltage. We will see in the next section that the behaviour is very different in interacting systems.

Finally, we find for the stationary tunneling current

$$
I_{r}=\frac{e}{h} \sum_{r^{\prime} \neq r} \int d \omega T_{r r^{\prime}}(\omega)\left[f_{r}(\omega)-f_{r^{\prime}}(\omega)\right]
$$

with the one-particle transmission probability given by

$$
T_{r r^{\prime}}(\omega)=\frac{M,{ }_{r}(\omega),{ }_{r^{\prime}}(\omega)}{(\omega-\epsilon-\operatorname{Re} \sigma(\omega))^{2}+(\operatorname{Im} \sigma(\omega))^{2}} .
$$

This formula agrees with the well-known Landauer-Büttiker formalism [7, 37] and is discussed in detail in [22]. In linear response, we have $e \bar{V}_{r}=$ $\mu+e \delta \bar{V}_{r}$ with $e \delta \bar{V}_{r} \ll T$, . This gives $I_{r}=\sum_{r^{\prime}} G_{r r^{\prime}}\left(\delta \bar{V}_{r}-\delta \bar{V}_{r^{\prime}}\right)$ with the conductance matrix given by the Breit-Wigner formula

$$
G_{r r^{\prime}}=-2 \pi \frac{e^{2}}{h} \frac{,^{r}, r^{\prime}}{,} \int d \omega \delta_{\Gamma}(\omega) f^{\prime}(\omega+\epsilon-\mu)
$$

where we have neglected the energy dependence of,$r(\omega)$. 
For $T \gg$, (incoherent or sequential tunneling limit), we obtain

$$
G_{r r^{\prime}}=-2 \pi \frac{e^{2}}{h} \frac{,^{r}, r^{\prime}}{,} f^{\prime}(\epsilon-\mu) \quad, \quad G_{r r^{\prime}}^{\max }=2 \pi \frac{e^{2}}{h} \frac{,^{r}, r^{\prime}}{,^{2}} \frac{1}{T},
$$

i.e. a symmetric line shape of the resonance around $\mu=\epsilon$ with exponential tails. With decreasing temperature the line width decreases $\sim T$ and the height of the resonance increases $\sim 1 / T$.

For $T \ll$, (coherent or resonant tunneling limit), we obtain

$$
G_{r r^{\prime}}=2 \pi \frac{e^{2}}{h} \frac{{ }^{r},{ }^{r^{\prime}}}{,} \delta_{\Gamma}(\epsilon-\mu) \quad, \quad G_{r r^{\prime}}^{\max }=\frac{e^{2}}{h} \frac{,^{r}, r^{\prime}}{(, / 2)^{2}},
$$

i.e. the line shape saturates at zero temperature to a lorentzian form reflecting the energy dependence of the transmission probability. For the special case of two reservoirs which couple symmetrically to the dot, the height of the resonance is given by the quantum conductance $e^{2} / h$. Compared to the incoherent limit we see that quantum fluctuations tend to suppress the conductance and broadens the line shape. The latter behaviour will also be obtained qualitatively in the interacting case described in the following sections. However, we will see that the line shape has no longer to be symmetrically, there can be logarithmic temperature or bias voltage dependences of peak position, peak height and broadening, and we will find interesting anomalies for the differential conductance as function of the bias voltage. All these features are completely absent in the noninteracting case, since the renormalization and broadening of the dot level have no interesting structure.

\subsection{INTERACTING QUANTUM DOT}

In this section we will study a more realistic and interesting case, namely the presence of two relevant excitation energies $\epsilon_{\sigma}=E_{s_{\sigma}}-E_{s_{0}}$, with $\sigma=\uparrow, \downarrow$, in the dot. This means that we consider two possible transitions when a particle tunnels into the dot. If the incoming electron has spin up or down we consider the transition $s_{0} \rightarrow s_{\uparrow}$ or $s_{0} \rightarrow s_{\downarrow}$, respectively. Due to spin conservation the corresponding Hamiltonian is given by

$$
H(t)=\sum_{k \sigma r} \epsilon_{k}^{r} a_{k \sigma r}^{\dagger} a_{k \sigma r}+\sum_{\sigma} \epsilon_{\sigma} \hat{P}_{s_{\sigma}}+\sum_{k \sigma r}\left(\bar{T}_{k}^{r}(t) a_{k \sigma r}^{\dagger} \hat{P}_{s_{0}, s_{\sigma}}+\text { h.c. }\right),
$$

where we have assumed spin independent tunneling matrix elements and used $\hat{P}_{s_{0}}=1-\sum_{\sigma} \hat{P}_{s_{\sigma}}$. Each reservoir line carries now a spin index in addition to the reservoir index. 
This model has a very interesting analog in the theory of strongly correlated fermions, namely the so-called infinite- $U$ Anderson impurity model in nonequilibrium which is described by the Hamiltonian

$$
H(t)=\sum_{k \sigma r} \epsilon_{k}^{r} a_{k \sigma r}^{\dagger} a_{k \sigma r}+\sum_{\sigma} \epsilon_{\sigma} c_{\sigma}^{\dagger} c_{\sigma}+U n_{\uparrow} n_{\downarrow}+\sum_{k \sigma r}\left(\bar{T}_{k}^{r}(t) a_{k \sigma r}^{\dagger} c_{\sigma}+h . c .\right),(
$$

with $U \rightarrow \infty$ being assumed to be the largest energy scale in the system. The role of the dot is here taken over by the role of a local impurity with one single spin $1 / 2$ state. $U$ is the on-site Coulomb repulsion and takes over the role of the charging energy. Since $U$ is assumed to be large, double occupancy of the impurity level is suppressed and only the three states $|0>,| \uparrow>$, and $|\downarrow\rangle$ are possible. They are identified with the states $\left|s_{0}\right\rangle$, $\left|s_{\uparrow}\right\rangle$, and $\left|s_{\downarrow}\right\rangle$, corresponding to the Hamiltonian (62), respectively. This gives $c_{\sigma} \equiv \hat{P}_{s_{0}, s_{\sigma}}$, and we can see that the two Hamiltonians are equivalent.

The significance of this equivalence lies in the fact that it is known from equilibrium theory that the Anderson model reveals a very interesting lowtemperature behaviour. For degenerate energies $\epsilon=\epsilon_{\uparrow}=\epsilon_{\downarrow}$ and in the Kondo regime $\epsilon \ll-$, , the system shows resonant transmission at zero temperature although the level position is far away from the Fermi level (defined at zero energy). The reason is that the transmission probability develops a Kondo resonance at the Fermi level for temperatures below the Kondo temperature $T_{K} \sim(U,)^{1 / 2} \exp (\pi \epsilon /),[28,39]$. The height of this resonance increases $\sim \ln \left(T_{K} / T\right)$ and saturates for very low temperatures. At zero temperature the Kondo resonance is decreasing when the level $\epsilon$ approaches -, from below since the system leaves the Kondo regime. However, for experimentally accessible temperatures, the Kondo resonance is only visible for $\epsilon \sim-$, due to the exponential dependence of the Kondo temperature on $\epsilon$. This is the cross-over from the Kondo regime to the mixed valence regime and corresponds roughly to the optimal value for the height of the resonance at the Fermi level.

The idea to test these features by measuring zero-bias anomalies of the differential conductance has a long history and many experiments have been performed [40]. The disadvantage here is that the current is measured through an ensemble of impurities and the control over physical parameters like coupling constants or impurity level positions is weak. Therefore the idea was formulated to test such features by measuring the conductance through quantum dots [41]. Various calculations were performed for the differential conductance as function of the bias voltage $[42,43]$ with the result of a zero-bias anomaly in the form of a maximum in the Kondo regime. It was predicted that the Kondo resonance splits by an applied bias and is shifted by Zeeman splitting [43]. The latter leads to a splitting of the zerobias maximum. These features have been observed experimentally by Ralph 
\& Buhrman [44]. They measured the differential conductance through single charge traps in a metallic quantum point contact. Although this system does not allow a controlled variation of the level position, the appearance of a zero bias maximum with a peak height varying logarithmically with temperature clearly demonstrates the mechanism of Kondo assisted tunneling. A detailed comparism of the line shape between experiment and theory can be found in Refs. $[17,45]$. The influence of external time dependent fields or bosonic environments was studied in Refs. $[16,17,46]$ with the result of side band anomalies in the differential conductance and pump effects. A closer investigation of the zero-bias anomaly reveals a cross-over of the zero-bias maximum to a zero-bias minimum by shifting the level position of the dot through the Fermi level [16, 17]. Further studies of the Kondo effect in quantum dots involve the AC-conductance in linear response [47] and Aharonov-Bohm oscillations [9].

To understand some of these results let us apply the resonant tunneling approximation. It can be evaluated analytically for the degenerate case which we will consider from now on. First we note that due to spin conservation the reduced density matrix of the dot is diagonal once it is diagonal at the initial time. The solution is identical to the one for the noninteracting dot, given by (54) and (55) for the transition rates and (57) and (58) for the tunneling current, but with an important change of the definition of $\gamma_{r}(\omega)=2 \gamma_{r}^{+}(\omega)+\gamma_{r}^{-}(\omega)$. The golden rule tunneling "in" rate $\gamma_{r}^{+}$has to be multiplied with a factor 2 since there are 2 possibilities for an electron to tunnel onto the dot. Adding the golden rule tunneling "out" rate $\gamma_{r}^{-}$, we obtain $\gamma_{r}$ which is an estimate for the inverse finite life-time of the dot excitation. This is expressed by the imaginary part of $\sigma(\omega)$ which is given by $\operatorname{Im} \sigma(\omega)=-\pi \sum_{r} \gamma_{r}(\omega)=-\frac{1}{2} \sum_{r},{ }_{r}(\omega)\left(1+f_{r}(\omega)\right)$. We see that the broadening depends now on the Fermi functions and is therefore energy, temperature and voltage dependent. When energy increases the broadening decreases, i.e. we expect quantum fluctuations to become weaker if we increase $\epsilon$.

From the Kramers-Kronig relation we have necessarily also a renormalization which is given by the real part of $\sigma(\omega)$. We obtain

$$
\begin{aligned}
\operatorname{Re} \sigma(\omega) & =\frac{1}{2 \pi} \sum_{r},{ }_{r}(\omega) \cdot \\
\cdot & {\left[\psi\left(\frac{1}{2}+\frac{\beta E_{C}}{2 \pi}\right)-\operatorname{Re} \psi\left(\frac{1}{2}+i \frac{\beta}{2 \pi}\left(\bar{V}_{r}-\omega\right)\right)+\pi \frac{\omega-\bar{V}_{r}}{2 E_{C}}\right], }
\end{aligned}
$$

where $\psi$ is the digamma function and we have chosen a lorentzian form for ${ }_{r}(\omega)={ }^{r} E_{C}^{2} /\left(\left(\omega-\bar{V}_{r}\right)^{2}+E_{C}^{2}\right)$. The cut-off will be of the order of the charging energy $E_{C}$ since we do not allow for two electron to tunnel 
onto the dot. For $T \ll\left|\bar{V}_{r}-\omega\right| \ll E_{C}$, the renormalization depends logarithmically on energy $\operatorname{Re} \sigma(\omega) \approx 1 /(2 \pi) \sum_{r},{ }^{r} \ln \left(E_{C} /\left|\bar{V}_{r}-\omega\right|\right)$. This leads to a logarithmic increase of the renormalization when $\omega$ approaches the effective potentials $e \bar{V}_{r}$ of the reservoirs. As a consequence the transmission probability (58) has a maximum near $\omega \approx e \bar{V}_{r}$ since there is a solution of $\omega-\epsilon-\operatorname{Re} \sigma(\omega)=0$ near these values. This indicates the occurence of the Kondo resonance and explains the splitting when the potentials $\bar{V}_{r}$ are not equal (see inset of Fig. 8a).

To illustrate the consequences for the current let us start with the incoherent limit $T \gg$, . In this case we can neglect the renormalization and the transmission probability is a sharp function around $\omega \sim \epsilon$. Neglecting the energy dependence of ${ }_{r}(\omega)$ we can replace the transmission probability in formula (57) by $T_{r r^{\prime}}(\omega) \rightarrow-2 \pi,{ }^{r},{ }^{r^{\prime}} / \operatorname{Im} \sigma(\epsilon) \delta(\omega-\epsilon)$ This gives for the conductance in linear response

$$
G_{r r^{\prime}}=-4 \pi \frac{e^{2}}{h} \frac{,^{r}, r^{\prime}}{\sum_{\bar{r}},{ }^{\bar{r}}(1+f(\epsilon-\mu))} f^{\prime}(\epsilon-\mu) .
$$

As expected the line shape is assymmetric since the broadening of $\epsilon$ depends on $V_{D}$. This result shows a clear difference to the noninteracting case where the line shape is symmetric. It shows up already in the high temperature regime and can be calculated also from the golden rule approach. The assymmetry was first predicted by Beenakker [30] but has never been identified experimentally.

In the coherent regime $T \ll$, , the real part of $\sigma(\omega)$ becomes important. As already mentioned above, the resonance of the transmission probability at the Fermi levels is only significant for $\bar{V}_{r}-\epsilon \sim$, since the Kondo temperature depends exponentially on $\bar{V}_{r}-\epsilon$. In this regime the relevant energy scale for the onset of quantum fluctuations is , . In Fig. 8 we show the differential conductance $G=d I / d V\left(I=I_{R}=-I_{L}\right)$ as function of the bias voltage $V=V_{L}-V_{R}$ for $\epsilon-\mu \sim-$, and $\epsilon \sim \mu$. Thereby we have chosen $V_{L}=-V_{R}=V / 2$ and used $e V_{D}=e \sum_{i=L, R, g}\left(C_{i} / C\right) V_{i}$ with symmetric capacitances $C_{L}=C_{R}$. This gives $e V_{D}=\left(C_{g} / C\right) e V_{g}$ independent of the bias voltage. For a low lying level a pronounced zero bias maximum is developed which is due to the fact that the Kondo resonances of the transmission probability at $\omega=\bar{V}_{r}, r=L, R$, are split by the bias voltage and decrease in magnitude (see inset of Fig. 8a). In contrast, for $\epsilon+V_{D}$ near the electrochemical potentials of the reservoirs, a zero bias minimum is observed although the Kondo resonances are absent. This is due to the fact that the nontrivial structure of the real part of $\sigma(\omega)$ is still present and influences the differential conductance always for $T \ll$, independent of whether the transmission probability shows Kondo resonances or not. The striking difference of the zero-bias anomaly for different values of $V_{D}$ or 


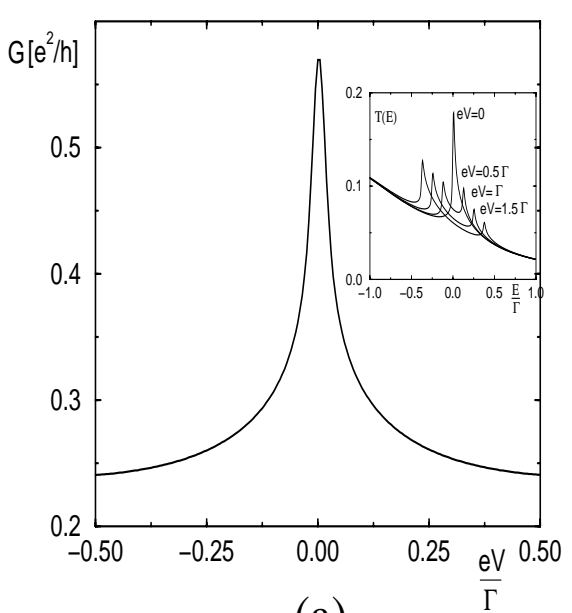

(a)

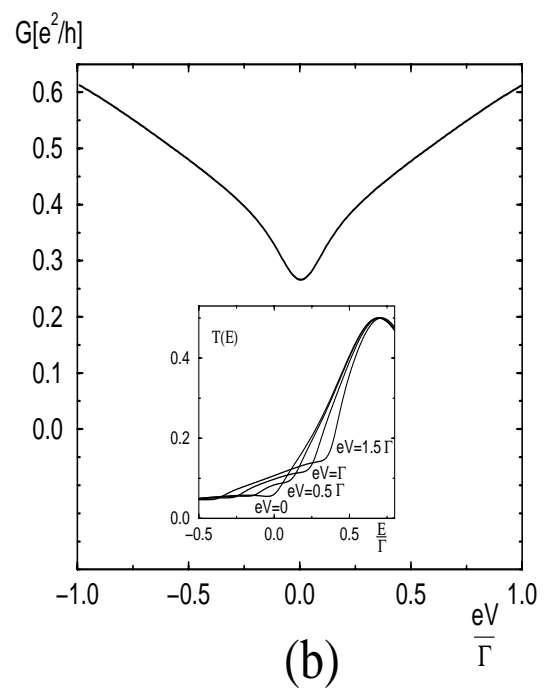

(b)

Figure 8. (a) The differential conductance vs. bias voltage for $\Gamma^{L}=\Gamma^{R}=\Gamma / 2 \Gamma$ $T=0.01 \Gamma \Gamma \mu=0 \Gamma \epsilon=-4 \Gamma$ and $E_{C}=100 \Gamma$. The curve shows a maximum at zero bias. Inset: increasing voltage leads to an overall decrease of the transmission probability in the range $|E|<e V$. (b) The differential conductance vs. bias voltage for $\Gamma^{L}=\Gamma^{R}=\Gamma / 2 \Gamma$ $T=0.05 \Gamma \Gamma V_{D}=0 \Gamma \epsilon=0$ and $E_{C}=100 \Gamma$. The curve shows a minimum at zero bias. Inset: increasing voltage leads to an overall increase of the transmission probability in the range $|E|<e V$.

$V_{g}$ motivates an interesting experiment which can only be performed with devices where the effective positions of the dot excitations can be varied by an external gate voltage.

\subsection{METALLIC ISLAND}

In this section we study the resonant tunneling approximation for the infinite- $Z$ metallic island. In addition, we assume that only one excitation energy $\Delta_{N}=E_{N+1}-E_{N}$ with $E_{N}=E_{C} N^{2}$ lies within the relevant energy range of the effective potentials $e \bar{V}_{r}=e V_{r}-e V_{D}$ of the reservoirs. This means that the charging energy $E_{C}$ is assumed to be much larger than temperature and bias voltage so that the other excitations are irrelevant. Without loss of generality we can set $N=0$.

In the absence of time-dependent fields and the heat bath, the Hamiltonian follows from (15) and (16)

$$
H(t)=\sum_{r=L, R, D ; k} \epsilon_{k}^{r} a_{k r}^{\dagger} a_{k r}+\Delta_{0} \hat{P}_{1}+\sum_{r=L, R ; k l}\left(\bar{T}_{k l}^{r}(t) a_{k r}^{\dagger} a_{l D} \hat{P}_{01}+\text { h.c. }\right),(66)
$$

where the time dependence of the coupling constants is only due to the 
static voltages $\bar{T}_{k l}^{r}(t)=T_{k l}^{r} \exp \left(i e \bar{V}_{r}\left(t-t_{0}\right)\right)$. This Hamiltonian looks very similiar to (53) where we considered a quantum dot with one excitation energy. However, the important difference here is that the vertex $a_{k r}^{\dagger} a_{l D}$ leads to bosonic contractions in the infinite- $Z$ case whereas in (53) we had to deal with fermionic contractions. Therefore, the resonant tunneling approximation does not turn out to be exact here. We obtain the same solution as in the fermionic case but with the replacements, ${ }_{r}(\omega) \rightarrow 2 D_{r}\left(\omega-e \bar{V}_{r}\right)$, $f_{r}^{ \pm} \rightarrow n_{r}^{ \pm}, \gamma_{r}^{ \pm} \rightarrow \alpha_{r}^{ \pm}$, and $\gamma_{r} \rightarrow \alpha_{r}=\alpha_{r}^{+}+\alpha_{r}^{-}$, where $D_{r}(\omega)=\pi \alpha_{0}^{r} \omega$ and $\alpha_{r}^{ \pm}=(1 / \pi) D_{r}\left(\omega-e \bar{V}_{r}^{0}\right) n_{r}^{ \pm}(\omega)$ follow from (33) and (40).

The tunneling current is given by (57) but the Fermi functions in this expression are replaced by Bose distributions. Using (58) together with the above mentioned replacements, and performing some elementary manipulations, we can rew rite the current as $I_{r}=e / h \sum_{r^{\prime}} \int d \omega T_{r r^{\prime}}^{F}(\omega)\left[f_{r}(\omega)-f_{r^{\prime}}(\omega)\right]$ where $T_{r r}^{F}$, is the transmission probability between the original Fermi reservoirs

$$
T_{r r^{\prime}}^{F}(\omega)=4 \pi^{2} \frac{\alpha_{r}(\omega) \alpha_{r^{\prime}}(\omega)}{\left(\omega-\Delta_{0}-\operatorname{Re} \sigma(\omega)\right)^{2}+(\operatorname{Im} \sigma(\omega))^{2}} .
$$

Renormalization and broadening effects are described by the real and imaginary part of $\sigma(\omega)=\int d \omega^{\prime} \sum_{r} \alpha_{r}\left(\omega^{\prime}\right) /\left(\omega-\omega^{\prime}+i \eta\right)$. We see that in contrast to the fermionic case the bosonic distribution functions $n_{r}^{ \pm}$occuring in $\alpha_{r}^{ \pm}$do not cancel in the sum $\alpha_{r}$. Like in the quantum dot case with two excitations, this gives rise to a broadening which depends on energy, temperature and voltage, and via Kramers Kronig to a nontrivial renormalization. Explicitly, we get $\operatorname{Im} \sigma(\omega)=-\sum_{r} D_{r}\left(\omega-e \bar{V}_{r}\right)\left(1+2 n_{r}(\omega)\right)$ and

$$
\begin{aligned}
\operatorname{Re} \sigma(\omega)= & -\frac{1}{\pi} \sum_{r} D_{r}\left(\omega-e \bar{V}_{r}\right) \cdot \\
& \cdot\left[\psi\left(\frac{E_{C}}{2 \pi T}\right)+\psi\left(1+\frac{E_{C}}{2 \pi T}\right)-2 \operatorname{Re} \psi\left(i \frac{\left|\omega-e \bar{V}_{r}\right|}{2 \pi T}\right)\right],
\end{aligned}
$$

where we have chosen a lorentzian form for $D_{r}(\omega)=\pi \alpha_{0}^{r} \omega E_{C}^{2} /\left(\omega^{2}+E_{C}^{2}\right)$. $\alpha_{0}^{r}$ is the dimensionless conductance of barrier $r$ defined after (33). For very low temperatures we get $\operatorname{Im} \sigma(\omega) \approx-\pi \sum_{r} \alpha_{0}^{r}\left|\omega-e \bar{V}_{r}\right|$ and $\operatorname{Re} \sigma(\omega) \approx$ $-2 \sum_{r} \alpha_{0}^{r}\left(\omega-\bar{V}_{r}\right) \ln E_{C} /\left|\omega-\bar{V}_{r}\right|$. The broadening is proportional to energy since the number of available states for tunneling on or off the island is also proportional to energy. In contrast to the interacting quantum dot in the previous section, the renormalization is zero for $\omega \sim \bar{V}_{r}$. Therefore, no additional resonances occur here for the transmission probability but we still have a logarithmic shift of the excitation energy $\Delta_{0}$.

The renormalization of $\Delta_{0}$ is determined by finding the maximum of the transmission probability (67) which is approximately determined by solving the self-consistent equation $\tilde{\Delta}_{0}=\Delta_{0}+\operatorname{Re} \sigma\left(\tilde{\Delta}_{0}\right)$. In a first approximation 
we use $\tilde{\Delta}_{0}$ for the value of $\omega$ inside the $\psi$-function of the real part of $\sigma$ given by (68). For $\omega \ll E_{C}$ we obtain $\omega-\Delta_{0}-\operatorname{Re} \sigma(\omega)=Z^{-1}\left(\omega-\tilde{\Delta}_{0}\right)$ with the renormalization factor $Z$ defined by

$$
Z^{-1}=1+\sum_{r} \alpha_{0}^{r}\left[\psi\left(\frac{E_{C}}{2 \pi T}\right)+\psi\left(1+\frac{E_{C}}{2 \pi T}\right)-2 \operatorname{Re} \psi\left(i \frac{\left|\tilde{\Delta}_{0}-e \bar{V}_{r}\right|}{2 \pi T}\right)\right] .
$$

Within this approximation the transmission probability reads

$$
T_{r r^{\prime}}^{F}(\omega) \approx 4 \pi^{2} \frac{\tilde{\alpha}_{r}(\omega) \tilde{\alpha}_{r^{\prime}}(\omega)}{\left(\omega-\tilde{\Delta}_{0}\right)^{2}+(\operatorname{Im} \tilde{\sigma}(\omega))^{2}},
$$

where $\tilde{\alpha}_{r}$ and $\tilde{\sigma}$ are defined as before but multiplied with $Z$. This can be interpreted as a renormalization of the dimensionless conductance $\tilde{\alpha}_{0}^{r}=$ $Z \alpha_{0}^{r}$. What we mean by renormalization becomes clear when we neglect the broadening in (70) which is described by the imaginary part of $\tilde{\sigma}$. This is justified if $\tilde{\alpha}_{0}^{r} \ll 1$. We obtain

$$
T_{r r^{\prime}}^{F}(\omega) \approx 4 \pi^{2} \frac{\tilde{\alpha}_{r}\left(\tilde{\Delta}_{0}\right) \tilde{\alpha}_{r^{\prime}}\left(\tilde{\Delta}_{0}\right)}{\sum_{\bar{r}} \tilde{\alpha}_{\bar{r}}\left(\tilde{\Delta}_{0}\right)} \delta\left(\omega-\tilde{\Delta}_{0}\right) .
$$

This is precisely the golden rule result for the transmission probability but with renormalized parameters.

In certain limits we can estimate the renormalized parameters. We take $V_{L}=-V_{R}=V, V_{D}=0$ (otherwise one has to shift the excitation energy $\Delta_{0}$ by $V_{D}$ ), and $\alpha_{0}^{L}=\alpha_{0}^{R}=\alpha_{0}$. If one of the energy parameters $\tilde{\Delta}_{0}, T$, or $e V$ is large compared to the other two ones but small compared to the charging energy, we obtain for the renormalization factor

$$
Z=\frac{1}{1+4 \alpha_{0} \ln \left(E_{C} / \max \left(\left|\tilde{\Delta}_{0}\right|, 2 \pi T,|e V| / 2\right)\right)} .
$$

We note that $\alpha_{0}$ is the dimensionless conductances of a single barrier. For the derivation we have used the asymptotic expansion $\psi(z)=\ln (z)$, valid for $|z| \rightarrow \infty$. The renormalized parameters follow from $\Delta_{0}=Z \Delta_{0}$ and $\tilde{\alpha}_{0}^{r}=Z \alpha_{0}^{r}$. These equations agree with the renormalization group results performed for the equilibrium case $V_{r}=0$ [48]. This shows that the leading logarithmic terms are included within the resonant tunneling approximation. However, we have achieved more than renormalization group here since we do not need all the approximative steps used so far. We can handle all intermediate regimes for the three energy parameters described before and can account for the broadening of the charge excitations by not neglecting the imaginary part of $\tilde{\sigma}$ in $(70)$. The latter can be estimated to be of the order $h / \tau \sim \operatorname{Im} \sigma\left(\tilde{\Delta}_{0}\right)$. Within the same limits discussed before 
this gives $h / \tau \sim \pi \tilde{\alpha_{0}} \max \left(\left|\tilde{\Delta}_{0}\right|, 2 T,|e V| / 2\right)$ (compare with the discussion in section 2.1). We see that broadening effects start to become important for $\tilde{\alpha}_{0}>0.1$ which means that they can only be enhanced by lowering the tunneling barriers. Renormalization effects become significant for $\max \left(\left|\tilde{\Delta}_{0}\right|, 2 \pi T,|e V| / 2\right)<E_{C} e^{-1 /\left(2 \alpha_{0}\right)}$. This means that they can be enhanced either by lowering the tunneling barriers or by lowering all the other energy parameters.

Let us demonstrate the influence of quantum fluctuations on the differential conductance as function of the gate voltage. Again we set $V_{L}=$ $-V_{R}=V / 2$ and $\alpha_{0}^{L}=\alpha_{0}^{R}=\alpha_{0}$. We study $G=d I / d V$, with $I=I_{R}=-I_{L}$, as function of $\Delta_{0}$ and set $V_{D}=0$ (equivalently we could study $G$ as function of $e V_{D}=\left(C_{g} / C\right) e V_{g}$ and keep $\Delta_{0}$ fixed $)$. We insert the transmission probability (70) including the broadening into the current formula. Using the result (72) for the renormalization factor, we find in the two limits $T \ll|e V|$ and $|e V| \ll T$ that the differential conductance at $\Delta_{0}=\tilde{\Delta}_{0}=0$ is given by

$$
2 G\left(\Delta_{0}=0\right) R_{T}=\frac{Z}{2}=\frac{1}{2} \frac{1}{1+4 \alpha_{0} \ln \left(E_{C} / \max (2 \pi T,|e V| / 2)\right)},
$$

where $R_{T}=R_{T}^{L}=R_{T}^{R}$ is the resistance of a single barrier. The golden rule result is $2 G\left(\Delta_{0}=0\right) R_{T}=1 / 2$ and corresponds to $1 / 2$ of the ohmic resistance since all the other excitation energies $\Delta_{N}(N \neq 0)$ are suppressed by the Coulomb blockade. We see that due to quantum fluctuations, the differential conductance is no longer a constant at the symmetry point but decreases logarithmically with bias voltage or temperature. We note that the qualitative effect of quantum fluctuations is again a suppression of the differential conductance like it was the case for quantum dots. It is not suprising that the differential conductance for the noninteracting quantum dot case saturates at low temperatures whereas it decreases for the metallic island since the temperature dependence of the golden rule results are already different for the two cases.

The broadening of the line shape of the differential conductance can be estimated by noting that the integral of $G\left(\Delta_{0}\right)$ over $\Delta_{0}$ is not influenced by quantum fluctuations and can be calculated to be $\int d \Delta_{0} G\left(\Delta_{0}\right) R_{T}=$ $(1 / 3)|e V|$ for $T \ll|e V|$, and $\int d \Delta_{0} G\left(\Delta_{0}\right) R_{T}=\left(\pi^{2} / 8\right) T$ for $|e V| \ll T$. Together with the value at the symmetry point we conclude that quantum fluctuations lead to a broadening that increases logarithmically with bias voltage or temperature if we measure $\Delta_{0}$ in units of $|e V|$ or $T$.

Both features, the logarithmic decrease of $G\left(\Delta_{0}=0\right)$ and the logarithmic increase of the broadening with bias voltage or temperature is demonstrated in Fig. 9. In Fig. 9b we furthermore observe a splitting of the resonance due to nonequilibrium effects and a shift of the individual resonances 


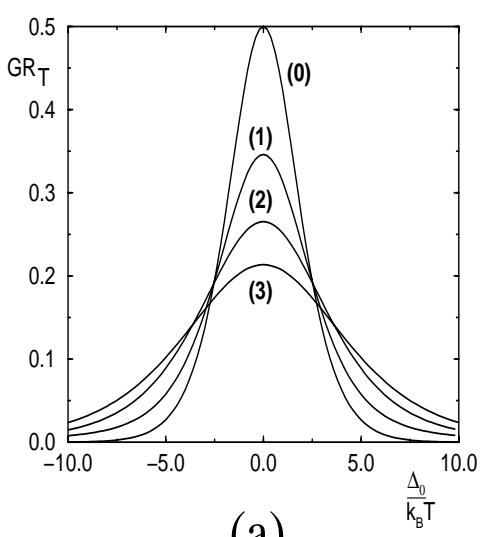

(a)

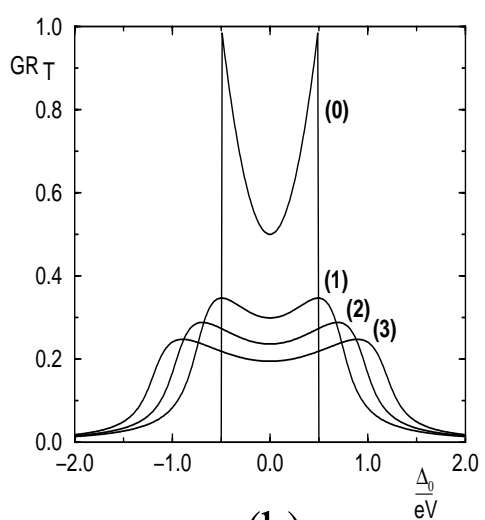

(b)

Figure 9. (a) The differential conductance in linear response ( $\mathrm{V}=0$ ) for the metallic island as a funciton of the excitation energy $\Delta_{0}$ normalized to the temperature $k_{B} T$ with $\alpha_{0}^{L}=\alpha_{0}^{R}=0.05$ and (1) $k_{B} T / E_{C}=0.1 \Gamma(2) k_{B} T / E_{C}=0.01 \Gamma(3) k_{B} T / E_{C}=0.001$. For comparison $\Gamma(0)$ shows the golden rule result $\Gamma$ which is independent of the temperature $k_{B} T$. (b) The differential conductance in nonlinear response for the metallic island as a function of the excitation energy $\Delta_{0}$ normalized to the transport voltage $e V$ with $\alpha_{0}^{L}=\alpha_{0}^{R}=0.05 \Gamma T=0$ and (1) $\mathrm{eV} / E_{C}=0.1 \Gamma(2) \mathrm{eV} / E_{C}=0.01 \Gamma(3) \mathrm{eV} / E_{C}=0.001$. For comparison $\Gamma(0)$ shows the golden rule result $\Gamma$ which is independent of the transport voltage $e V$.

due to quantum fluctuations. The logarithmic decrease of $G\left(\Delta_{0}=0\right)$ has been observed experimentally [10] with a good fit to the theoretical predictions [49].

\section{Conclusions}

Within this paper we have analysed a very fundamental problem of statistical mechanics, namely the interaction between a large environment and a small mesoscopic system. To describe experimentally realizable systems, we concentrated on particle exchange via tunneling and energy exchange by considering a fluctuating voltage. For the environment we have chosen metallic electronic reservoirs with different electrochemical potentials, and the system was realized by a strongly interacting quantum dot. Macroscopic systems being in equilibrium with large particle reservoirs are described by a grandcanonical ensemble. For a mesoscopic system we have to consider the following new aspects. First, the energy scale associated with the coupling between system and environment can be so large that quantum fluctuations lead to a complete deviation from a grandcanonical ensemble. In macroscopic systems, the coupling to the environment is always a surface effect which can be negleted in the thermodynamic limit. Second, the en- 
ergy scale characterizing the distance between the one-particle excitation energies of the system can be so large that the discreteness of the density of states becomes visible on experimentally controllable voltage scales. This demands the consideration of finite size effects and strong capacitive interactions. Third, the nonequilibrium stationary state induced by different electrochemical potentials on the reservoirs can no longer be described by a local equilibrium distribution. Therefore, we have aimed at presenting a nonequilibrium theory which provides a nonperturbative analysis in the coupling between an environment and a strongly correlated finite system.

We have demonstrated that the measurement of the differential conductance $G$ as function of the gate voltage $V_{g}$ or the bias voltage $V$ can reveal all aspects decribed above. The discreteness of the dot excitation spectrum leads to resonances in $G\left(V_{g}\right)$ separated by the sum of level spacing and charging energy. Strong nonequilibrium effects can be observed by comparing the line shape of an individual peak for $V=0$ and $T=0$. E.g. for a metallic island we have shown these two cases in Fig. 9 where a splitting of the resonance occurs at finite bias voltage. Quantum fluctuations set on by lowering temperature or increasing tunneling. Without spin degeneracies, they lead to a renormalization and broadening of the excitation energies of the island. Whereas for noninteracting systems the effects on $G\left(V_{g}\right)$ are already well-known from Landauer-Büttiker theory, the presence of interactions can lead to an anomalous temperature dependence of height, broadening or position of the resonances. This is demonstrated in Fig 9 for the case of metallic islands. For spin degenerate excitation energies quantum fluctuations can create zero bias anomalies of $G(V)$ at fixed gate voltage as is demonstrated in Fig. 8. They can occur in the form of zero bias maxima or minima dependent on the postion of the excitation energies relative to the electrochemical potentials of the reservoirs. Due to the enormous variety of possible arrangements of island systems and the experimental progress in realizing such devices, we expect that future research will reveal many more motivations for studying quantum fluctuations induced by strong coupling between mesoscopic systems and particle reservoirs.

\section{Acknowledgements}

It is a pleasure to thank all my collaborators C. Bruder, R. Fazio, M. Hettler, J. König, J. Schmid and G. Schön. This work was supported by the Deutsche Forschungsgemeinschaft through Sonderforschungsbereich 195.

\section{References}

1. D.V. Averin and K.K. LikharevTin Mesoscopic Phenomena in solidsTedited by B. 


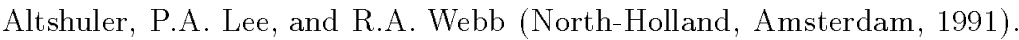

2. P.L. McEuen and L.P. Kouwenhoven To be published in Nano-Science and Technology $\Gamma$ G. Timp ed. (AIP Press $\Gamma$ New York).

3. M. A. KastnerTRev. Mod. Phys. 64Г849 (1992); Physics Today 46Г24 (1993).

4. H. Grabert and M.H. Devoret $\Gamma$ Single Charge Tunneling NNATO ASI Series $\Gamma$ Vol.294 (New York TPlenum Press 1992).

5. G. SchönT Single-Electron Tunneling $\Gamma$ to be published in Quantum Transport and Dissipation $\Gamma$ Chapter $2 \Gamma$ eds. T. Dittrich $\Gamma$ P. Hänggi $\Gamma$ G. Ingold $\Gamma B$. Kramer $\Gamma$ G. Schön $\Gamma$ and W. ZwergerT(VCH Publishers).

6. Z.Phys.B-Condensed Matter 85.

7. M. BüttikerГPhys. Rev. Lett. 57Г1761 (1986); Phys. Rev. B46Г12485 (1992).

8. D.V. Averin and Y.V. NazarovTPhys. Rev. Lett. 65 (1990) 2446; ibid. in Ref. [4].

9. C. BruderГR. FazioTand H. SchoellerГPhys. Rev. Lett. 76Г114 (1995).

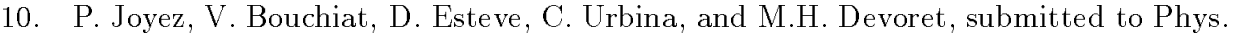
Rev. Lett.

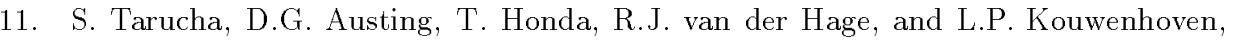
Phys. Rev. Lett. 77Г3613 (1996).

12. D.C. RalphГC.T. Black and M. TinkhamTPhys. Rev. Lett. 74Г3241 (1995); D.C.

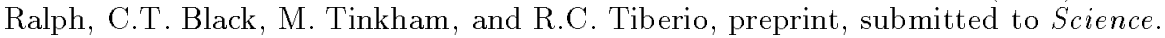

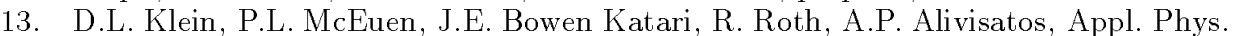
Lett. 68Г2574 (1996).

14. H. Schoeller and G. SchönTPhys. Rev. B50Г18436 (1994).

15. J. König ГH. Schoeller and G. SchönГEurophys. Lett. 31Г31 (1995).

16. J. König ГH. Schoeller and G. SchönГPhys. Rev. Lett. 76Г1715 (1996).

17. J. König ГJ. SchmidГH. SchoellerГ and G. SchönГPhys. Rev. B54Г16820 (1996).

18. R.P. Feynman and F.L. VernonT Ann. Phys. (N.Y.) 24 (1963) 118.

19. A. O. Caldeira and A. J. LeggettTPhysica A $121 \Gamma 587$ (1983); Ann. Phys. (N.Y.) 149Г374 (1983); 153Г445(E) (1983).

20. U. Weiss $\Gamma$ Quantum Dissipative Systems $\Gamma$ Series in Modern Condensed Matter Physics TVol. 2 (World Scientific $\Gamma 1993$ ).

21. U. EckernГG. Schön and V. AmbegaokarГPhys. Rev. B30Г6419 (1984); G. Schön and A.D. ZaikinTPhys. Rep. 198Г237 (1990).

22. M. BüttikerГIBM J. Res. Dev. 32Г317 (1988).

23. D. Pfannkuche and S.E. UlloaГPhys. Rev. Lett. 74Г1194 (1995).

24. K. Jauregui W. HäuslerГ D. Weinmann $\Gamma$ and B. Kramer $\Gamma$ Phys. Rev. 53 R1713 (1996).

25. N.S. WingreenГK.W. JacobsenTand J.W. WilkinsГPhys. Rev. Lett. 61Г1396 (1988); Phys. Rev. B40Г11834 (1989); L.I. Glazman and R.I. ShekhterTZh. Eksp. Teor. Fiz. 94Г292 (1988 [sov. Phys. JETP 67Г163 (1988)]; M. JonsonГPhys. Rev. B39Г5924 (1989).

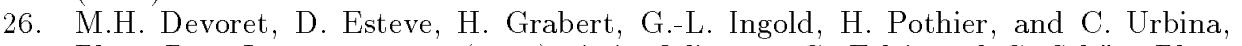

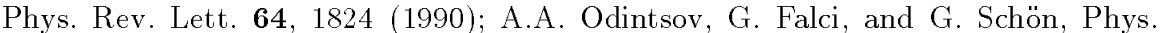

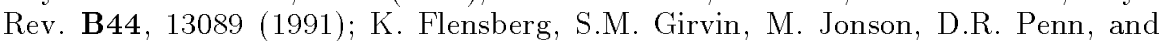

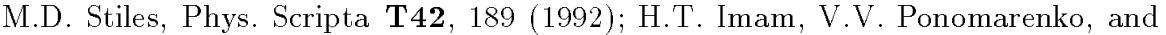
D.V. AverinTPhys. Rev B50Г18288 (1994).

27. G. Ingold and Yu.V. NazarovTin Ref. [4].

28. G.D. MahanT Many-Particle Physics $\Gamma($ Plenum T1990).

29. P.K. Tien and J.R. GordonГPhys. Rev. 129Г647 (1963).

30. C.W.J. BeenakkerГPhys. Rev. B44Г1646 (1991).

31. Y. MeirTN.S. Wingreen Tand P.A. LeeГPhys. Rev. Lett. 66 Г3048 (1991)

32. I.O. Kulik and R.I. SchekhterTSov. Phys. JETP 41 (1975) 308; L.I. Glazman and K.A. MatveevГ Pis'ma Zh. Eksp. Teor. Fiz. 48Г 403 (1988)Г[JETP Lett 48Г 445 (1988)]; H. van HoutenTC.W.J. BeenakkerT and A.A.M. StaringT in Ref.[4]; D.V. AverinГA.N. KorotkovГand K.K. LikharevГPhys. Rev. B44Г6199 (1991).

33. D. WeinmannTW. Häusler TW. PfaffГВ. KramerTand U. WeissTEurophys. Lett. $26 \Gamma$ 
467 (1994); W. HäuslerГ Ann. Physik 5T401 (1996).

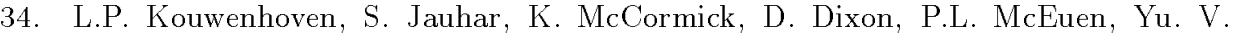
NazarovT N.C. van der VaartTand C.T. FoxonT Phys. Rev. B50Г2019 (1994); I.A. Devyatov and K.K. LikharevГPhysica B194-196Г1341 (1994).

35. C. Bruder and H. SchoellerГ Phys. Rev. Lett. 72Г1076 (1994).

36. C.A. Stafford ГPhys. Rev. Lett. 77Г2770 (1996).

37. R. LandauerГIBM J. Res. Dev. 1Г233 (1957);

38. C. CaroliГR. CombescotГP. Nozières and D. Saint-JamesГJ.Phys. C4Г916 (1971);

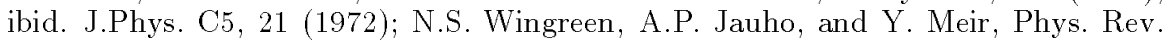
B48Г8487 (1993).

39. N.E. BickersTRev. Mod. Phys. 59Г845 (1987); A.C. HewsonT The Kondo Problem to Heavy Fermions (Cambridge University PressT1993).

40. J. AppelbaumГPhys. Rev. Lett. $17 \Gamma 91$ (1966); A.G.M. JansenГA.P. van GelderГP.

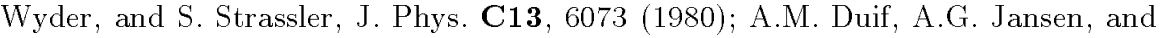
P. WyderГ J.Phys.: Cond. Matt. 1Г3157 (1989); E.L. WolfT Principles of Electron Tunneling Spectroscopy (Oxford Univ. PressTNew YorkT1989)ГChap. 8.

41. T.K. Ng and P.A. LeeTPhys. Rev. Lett. 61Г1768 (1988); L.I. Glazman and M.E. RaikhГPis'ma Zh. Eksp. Teor. Fiz. 47Г378 (1988) [JETP Lett. 47 T452 (1988)]; A. KawabataГJ. Phys. Soc. Japan 60Г3222 (1991).

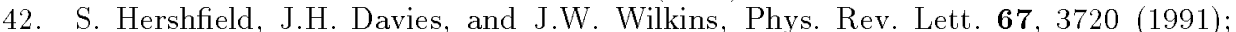
Phys. Rev. B46Г 7046 (1992); T.K. NgT Phys. Rev. Lett. 70Г 3635 (1993); A.L.

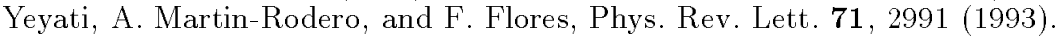

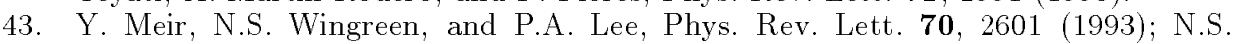
Wingreen and Y. MeirГPhys. Rev. B49Г11040 (1994).

44. D.C. Ralph and R.A. BuhrmanTPhys. Rev. Lett. 72Г3401 (1994).

45. N. Sivan and N.S. Wingreen Phys. Rev. B54Г11622 (1996).

46. M.H. Hettler and H. SchoellerГ Phys. Rev. Lett. 74Г4907 (1995).

47. T.K. NgГPhys. Rev. Lett. 76Г 487 (1996).

48. K.A. MatveevTSov. Phys. JETP $72 \Gamma 892$ (1991); G. FalciГG. Schön and G.T. Zi manyi Phys. Rev. Lett. $74 \Gamma 3257$ (1995).

49. J. König $\Gamma$ H. Schoeller $\Gamma$ and G. Schön $\Gamma$ submitted to Phys. Rev. Lett. $\Gamma$ condmat/9702196. 